\title{
WAVELET TRANSFORMS AND THEIR APPLICATIONS TO TURBULENCE
}

\section{Marie Farge}

LMD-CNRS Ecole Normale Supérieure, 24, rue Lhomond, 75231 Paris Cedex 5, France

KFY WORDS: orthogonal bases, turbulence

\section{INTRODUCTION}

Wavelet transforms are recent mathematical techniques, based on group theory and square integrable representations, which allows one to unfold a signal, or a field, into both space and scale, and possibly directions. They use analyzing functions, called wavelets, which are localized in space. The scale decomposition is obtained by dilating or contracting the chosen analyzing wavelet before convolving it with the signal. The limited spatial support of wavelets is important because then the behavior of the signal at infinity does not play any role. Therefore the wavelet analysis or synthesis can be performed locally on the signal, as opposed to the Fourier transform which is inherently nonlocal due to the space-filling nature of the trigonometric functions. Wavelet transforms have been applied mostly to signal processing, image coding, and numerical analysis, and they are still evolving.

So far there are only two complete presentations of this topic, both written in French, one for engineers (Gasquet \& Witomski 1990) and the other for mathematicians (Meyer 1990a), and two conference proceedings, the first in English (Combes et al 1989), the second in French (Lemarié 1990a). In preparation are a textbook (Holschneider 1991), a course (Daubechies 1991), three conference proceedings (Meyer \& Paul 1991, Beylkin et al 1991b, Farge et al 1991), and a special issue of IEEE Transactions 
on Information Theory (Daubechies et al 1991), which are all written in English.

Therefore, I assume that the reader is not yet familiar with this topic and give a general presentation of both the continuous wavelet transform and the discrete wavelet transform, in a manner as complete and detailed as possible, to provide the reader with the basic information with which to start using these transforms. In this spirit I will discuss the choice of the wavelct, which varics according to its application, and point out pitfalls to be avoided in the interpretation of wavelet transform results.

Since most of the existing work has so far been of an exploratory character and thus cannot be held as representative of the possible impact of wavelets on fluid mechanics, only brief reference will be made to papers dealing with applications. I shall also present several new diagnostics, all based on wavelet coefficients, which may be useful to analyze, model, or compute turbulent flows.

\section{THE NEED FOR A SPACE-SCALE DECOMPOSITION OF TURBULENT FLOWS}

In the field of turbulence, one may feel uneasy about the fact that we have two different pictures of turbulence, depending on the side of the Fourier transform from which we perceive it. On the one hand, if we look at the Fourier spectral space, we have a theory that assumes the existence of an energy cascade between the different excited wavenumbers of the flow. It predicts the universality of the Fourier energy spectrum in the inertial range, namely for wavenumbers larger than those corresponding to the integral scales at which the flow is excited and smaller than those corresponding to the dissipative scales where all instabilities are damped. In this Fourier space approach the direct numerical simulation of a turbulent flow requires a number of resolved Fourier wavenumbers which scales as $\mathrm{Re}$ for two-dimensional flows and as $\mathrm{Re}^{9 / 4}$ for three-dimensional flows ( $\mathrm{Re}$ being the Reynolds number characteristic of the flow turbulence). On the other hand, if we look at the physical space, we must admit a lack of general theory. Still, we have a large amount of evidence, both experimental (Townsend 1956, Kline et al 1967, Laufer 1975) and numerical (Basdevant et al 1981, McWilliams 1984, Kim et al 1987), for the presence of coherent structures in turbulent flows. They correspond to the condensation of the vorticity ficld into organized patterns, which contain most of the energy - or enstrophy in dimension two- of the flow and where nonlinearity is reduced, or even cancelled when the coherent structures are axisymmetric. These coherent structures seem to play an important, but not yet well understood, dynamical role. We can ask the following ques- 
tions. Are there some elementary coherent structures? Do their mutual interactions have a universal character? Is it possible to compute the flow evolution with a reduced number of degrces of freedom rclative to the very large number of Fourier components otherwise necessary? This reduction could correspond to a projection of Navier-Stokes equations on those coherent structures or on some related functional bases well localized in physical space.

Being very uncomfortable with these two separate descriptions of turbulence, I was immediately enthusiastic when, in 1984, A. Grossmann told me about the wavelet transform theory he was developing from Morlet's original ideas. His theory had the promise of a unified approach which could reconcile these two descriptions and allow us to analyze a turbulent flow in terms of both space and scale at once, up to the limits of the uncertainty principle. Another reason, rather naive, for the immediate appeal of wavelets was the fact that the Morlet wavelet evoked to me the shape of Tennekes and Lumley's eddy (Tennekes \& Lumley 1972) proposed to model turbulence, and of some coherent structures whose existence had been conjectured by Ruelle (D. Ruelle, personal communication, 1983) and then observed by Basdevant in his numerical simulations of two-dimensional flows (Basdevant \& Couder 1986). Indeed, it seems much better to decompose a turbulent field into such localized oscillations of finite energy as wavelets, rather than into space-filling trigonomctric functions which do not belong to the $L^{2}\left(\mathbf{R}^{n}\right)$ functional space and therefore are not of finite energy.

In the context of turbulence, the wavelet transform may yield some elegant decompositions of turbulent flows (Section 5.1). The continuous wavelet transform offers a continuous and redundant unfolding in terms of both space and scale, which may enable us to track the dynamics of coherent structures and measure their contributions to the energy spectrum (Section 5.2). The discrete wavelet transform allows an orthonormal projection on a minimal number of independent modes which might be used to compute or model the turbulent flow dynamics in a better way than with Fourier modes (Section 5.3).

\section{WAVELET TRANSFORM PRINCIPLES \\ 2.1 History}

The wavelet transform originated in 1980 with Morlet, a French research scientist working on seismic data analysis (Morlet 1981, 1983; Goupillaud et al 1984), who then collaborated with Grossmann, a theoretical physicist from the CNRS in Marseille-Luminy. They developed the geometrical formalism of the continuous wavelet transform (Grossmann et al 1985, 
1986, 1987, 1989; Grossmann 1988; Grossmann \& Morlet 1984, 1985; Grossmann \& Paul 1984; Grossmann \& Kronland-Martinet 1988) based on invariance under the affine group - namely translation and dilationwhich allows the decomposition of a signal into contributions of both space and scale (Section 3.1). In particular the continuous wavelet transform is well suited for analyzing the local differentiability of a function, and for detecting and characterizing its possible singularities (Holschneider 1988b; Jaffard 1989a; Arnéodo et al 1988; Holschneider \& Tchamitchian 1989; Mallat \& Hwang 1990; Jaffard 1991a,b). It is also useful for signal processing, in particular with the "skeleton" technique (Escudié \& Torrésani 1989, Tchamitchian \& Torrésani 1991, Delprat et al 1991) which allows the extraction of the modulation law of a complex signal, assuming some stationary phase hypothesis. The continuous wavelet transform has been extended to $n$ dimensions by Meyer (1985) and then by Murenzi using rotation, in addition to dilation and translation (Murenzi 1989, 1990; Antoine et al 1990,1991$)$. Murenzi is presently extending it to $n$ dimensions plus time (Duval-Destin \& Murenzi 1991). The wavelet transform then works as a "microscope," discriminating different scales in an $n$-dimensional field, and as a "polarizer," separating the different angular contributions of the signal.

When, in 1985, Meyer read Morlet and Grossmann's work, he recognized Calderon's identity (Calderon 1964) behind the admissibility condition and the reconstruction formula of the continuous wavelet theory (Section 3.1). He then collaborated with Grossmann and Daubechies (Daubechies et al 1986) to select a discrete subset of the continuous wavelet space, chosen in such a way that it constitutes a quasi-orthogonal complete set of $L^{2}\left(\mathbf{R}^{n}\right)$, called a wavelet frame (Section 4.1). Complementary to this, Morlet and Grossmann had previously defined an interpolation formulabased on the reproducing kernel property of the continuous wavelet transform (Section 3.2) - which recovers the whole space of continuous wavelet coefficients from the coefficients of a discrete subset, such as a wavelet frame (Grossman \& Morlet 1985). Meyer then tried to prove that, even if wavelet decomposition behaves in some sense as an orthogonal basis of $L^{2}\left(\mathbf{R}^{n}\right)$, there could not be any true orthogonal basis constructed with regular wavelets. The Haar orthogonal basis (Haar 1909) was well-known, but the lack of regularity of the functions it uses creates problems for decomposing smooth functions, whose Haar coefficients wotild only decay very slowly at infinity. Meyer was therefore surprised to discover an orthogonal basis (Section 4.2) built from a regular wavelet (Meyer 1986, 1987a,b, 1988). He later extended it to the $n$-dimensional case in collaboration with his student Lemarié (Lemarié \& Meyer 1986). In 1987, Meyer (1988, 1989a,b,c, 1990a,b,c) and Mallat (1988) introduced the con- 
cept of multiresolution analysis, which is very similar to the Quadratic Mirror Filters technique (Esteban \& Galand 1977) defined in digital processing and computer vision. This approach gives a general method for building orthogonal wavelet bases and leads to the implementation of fast wavelet algorithms (Mallat 1989a,b). Since then many other orthogonal wavelet bases have been found, among them: the Battle-Lemarié wavelet (Battle 1987, 1988; Lemarié 1988). which uses exponentially decreasing spline functions, the discrete orthogonal bases of Rioul $(1987,1991)$, and the compactly supported and regular wavelets of Daubechies $(1988,1989)$, built by iterating some discrete filters.

From today's point of view we can recognize, a posteriori, that several aspects of wavelet theory were already present, more or less explicitly, in many fields, such as image processing (Granlund 1978), because this kind of decomposition is indeed very natural. This is particularly clear for the Strömberg orthogonal basis (Strömberg 1981) used in functional analysis and for the hicrarchical basis of Zimin proposed to model turbulence (Zimin 1981). Today wavelet theory is a new and rapidly evolving mathematical techniquc, which has established similarities between various methods that were independently developed in different fields, from functional analysis to signal processing, and gives them a common theoretical framework.

\subsection{Definition}

What are the necessary ingredients of the wavelet transform?

ADMissibicity To be called a "wavelet," the analyzing function should be admissible (Section 3.1), which, for an integrable function, means that its average should be zero. This requirement excludes, for instance, functions used in Karhunen-Loève-also called Proper Orthonormal Decompositions (Lumley 1981, Aubry et al 1988) - which are not of zero mean value.

SIMILARITY The scale decomposition should be obtained by the translation and dilation of only one "mother" function. All analyzing wavelets should therefore be mutually similar, namely scale covariant with one another, in particular they should have a constant number of oscillations (Section 3.1). Thus this dilation procedure allows an optimal compromise in view of the uncertainty principle: The wavelet transform gives very good spatial resolution in the small scales and very good scale resolution in the large scales (Figure 1). This similarity condition excludes the windowed Fourier transform of Gabor (1946), whose scale decomposition is based on a family of trigonometric functions exhibiting increasingly many oscil- 

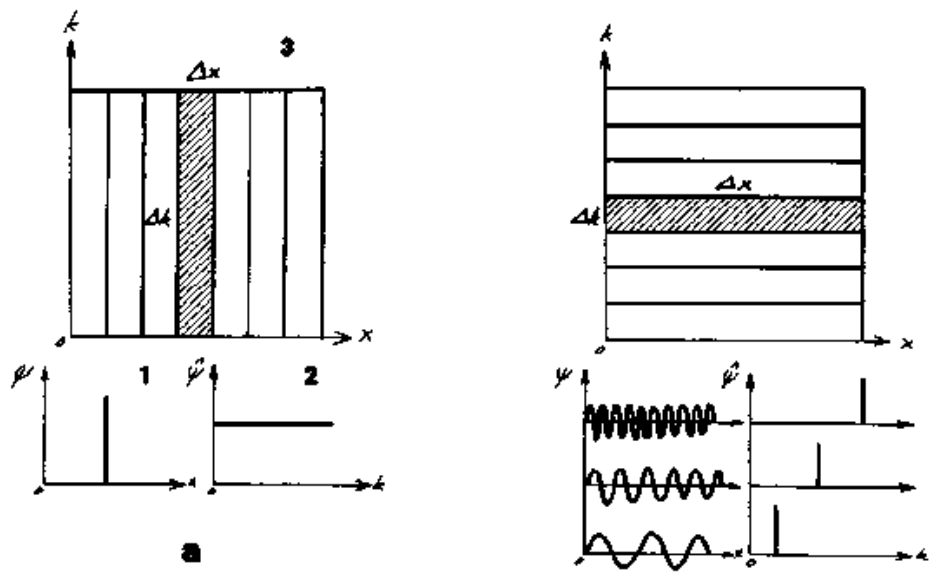

b
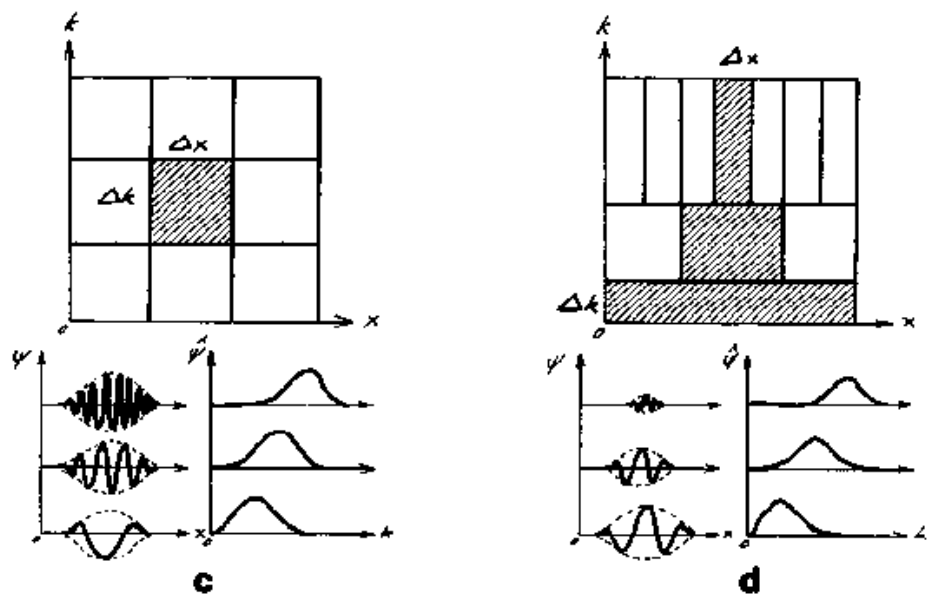

Figure I Phase space associated with different transforms. The uncertainty principle imposes phase-space "atoms" such that $\Delta x \cdot \Delta k \geq 2 \pi$. 1. Analyzing function in physical space, 2. analyzing function in Fourier space, and 3 . information cell $\Delta x-\Delta k$ in phase space. (a) Shannon sampling, (b) Fourier transform, (c) Gabor transform, (d) Wavelet transform.

lations in a window of constant size. In this case the spatial resolution in the small scales and the range in the large scales are limited by the size of the window (Figure 1).

INVER TIBILITY There should be at least one reconstruction formula for recovering the signal exactly from its wavelet coefficients and for allowing the computation of energy or other invariants directly from them (Sections 3.1 and 4.2 ). This precludes passband filtering techniques, renamed today 
"Fourierlets," which do not give an exact reconstruction formula for synthesizing the signal from its spectral coefficients.

REGULARITY In practice the wavelet should also be well localized on both sides of the Fourier transform, namely it should be concentrated on some finite spatial domain and be sufficiently regular. Indeed, there also exist regular wavelets that vanish outside a domain of compact support (Section 4.2). This additional regularity requirement excludes all discontinuous functions such as those used in the Haar orthogonal decomposition (Haar 1909).

CANCELlATIONS For some applications, in particular turbulent signal analysis, the wavelet should not only be of zero mean value (admissibility condition), but should also have some vanishing high-order moments (Section 3.1). This requirement, which eliminates the most regular (polynomial) part of the signal, allows the study of its high-order fluctuations and possible singularities in some high-order derivatives. In this case, the wavelet coefficients will be very small in the regions where the function is as smooth as the order of cancellation and the wavelet transform will only react to the higher order variations of the function.

\subsection{Comparison with the Fourier Transform}

Since Fourier's work on heat theory, the most commonly used basis functions in physics have been the trigonometric functions, because they constitute an orthogonal basis of $L^{2}(0,2 \pi)$, the functional space of square integrable functions. Thus they allow the decomposition of any function $f(x) \in L^{2}(0,2 \pi)$ into a linear combination of Fourier vectors, defined by their Fourier coefficients $\hat{f}(k)=\left\langle e^{i k x} \mid f(x)\right\rangle$. Unfortunately the trigonometric functions oscillate forever and therefore the information content of $f(x)$ is completely delocalized among all the spectral coefficients $\hat{f}(k)$. Indeed the Fourier transform does not lose information about $f(x)$, but instead "spreads" it away; it is then very difficult, or even impossible, as soon as there is some computational noise, to study the properties of $f(x)$ from those of $f(k)$. I et us for instance take the case of a function that is smooth everywhere except at a few singular points. The positions of the singularities are related to the phase of all the Fourier coefficients. Therefore there is no way to localize the singularities in Fourier space and the only solution will be to reconstruct $f(x)$ from $\hat{f}(k)$. Similarly, this function $f(x)$ will have a power-law spectrum so that the modulus of the Fourier coefficients will scale as $k$. This indicates that $f(x)$ is globally nonregular with singularities of at most exponent $\alpha$. Unfortunately, we have then lost the essential information, namely the fact that $f(x)$ is regular everywhere except at a few singular points. If, for instance, these singular points are 
due to experimental errors, we will not be able to filter them out because they have affected all the Fourier coefficients.

In contrast to the Fourier transform, the wavelet transform keeps the locality present in the signal and allows the local reconstruction of a signal. It is then possible to reconstruct only a portion of it or only its local contributions to a given range of scales. In fact, there is a relationship between the local behavior of a signal and the local behavior of its wavelet coefficients. For instance, if a function $f(x)$ is locally smooth, the corresponding wavelet coefficients will remain small, and if $f(x)$ contains a singularity, then in its vicinity the wavelet coefficients' amplitude will increase drastically (Section 3.2). Likewise the wavelet series of $f(x)$ converges locally to $f(x)$, even if $f(x)$ is a distribution (in this case the order of the distribution should not exceed the regularity of the analyzing wavelet). By "locally" we mean that, for reconstructing a portion of a signal, it is only necessary to consider the wavelet coefficients belonging to the corresponding subdomain of the wavelet space, the so-called influence cone (Section 3.2). Consequently, the wavelet transform is very robust for reconstruction. Indeed, if the wavelet coefficients are occasionally subject to errors, this will only affect the reconstructed signal locally near the perturbed positions, while the Fourier transform would spread out the errors everywhere in the reconstructed signal. The Fourier transform is also particularly sensitive to phase errors, due to the alternating character of the trigonometric series. This is not the case for wavelet transforms. It is even possible to correct the errors present in the continuous wavelet coefficients, thanks to the built-in redundancy of the continuous wavelet transform duc to its reproducing kernel property (Section 3.1).

In fact the wavelet transform is not intended to replace the Fourier transform, which remains very appropriate, for instance, in the study of harmonic signals or when there is no need for local information. Let us also mention that the Fourier transform plays a role in the admissibility condition defining wavelets (Section 3.1) and in the construction of discrete filters used in multiresolution analysis (Section 4.2). In practice the Fourier transform may be thought of as imbedded into the wavelet transform, because it is, to first approximation, possible to compute the Fourier spectrum of a signal by summing its wavelet coefficients over all positions scale by scale (Section 5.2).

\section{THE CONTINUOUS WAVELET TRANSFORM}

\subsection{Analysis and Synthesis}

WAVELET DEFINITION The only constraint imposed on a function $\psi(\mathbf{x})$, real or complex valued, in order to be a wavelet is the admissibility condition which requires: 


$$
C_{\psi}=(2 \pi)^{n} \int_{\mathbb{R}^{n}}|\hat{\psi}(\mathbf{k})|^{2} \frac{d^{n} \mathbf{k}}{|\mathbf{k}|^{n}}<\infty
$$

with

$$
\hat{\psi}(\mathbf{k})=(2 \pi)^{-n} \int_{\mathbb{R}^{n}} \psi(\mathbf{x}) e^{-\sqrt{-1} \mathbf{k} \cdot \mathbf{x}} d^{n} \mathbf{x},
$$

$n$ being the number of spatial dimensions. If $\psi(\mathbf{x})$ is integrable this actually implies that it has zero mean:

$$
\int_{\mathbf{R}^{n}} \psi(\mathbf{x}) d^{n} \mathbf{x}=\mathbf{0} \quad \text { or } \quad \dot{\psi}(|\mathbf{k}|=0)=0 .
$$

In practice the wavelet should also be well-localized in both physical and Fourier spaces. If one wants to study the behavior of the $M$ th derivative of $f(\mathbf{x})$, the wavelet should have cancellations up to order $M$, in order that it does not react to the lower-order variations of $f(\mathbf{x})$, namely we should have:

$$
\int_{\mathbf{R}^{n}} \psi(\mathbf{x}) \mathbf{x}^{m} d^{n} \mathbf{x}=0
$$

for all $m \leq M$.

WAVELET ANALYSIS From this function $\psi$, the so-called mother wavelet, we generate the family of continuously translated, dilated, and rotated wavelets:

$$
\psi_{\mathrm{N} \cdot \theta}(\mathbf{x})=l^{-n / 2} \psi\left[\Omega^{-1}(\theta) \frac{\mathbf{x}-\mathbf{x}^{\prime}}{l}\right]
$$

with $l \in \mathbf{R}^{+}$as the scale dilation parameter corresponding to the width of the wavelet and $\mathbf{x}^{\prime} \in \mathbf{R}^{n}$ as the translation parameter corresponding to the position of the wavelet; $l$ and $\mathbf{x}^{\prime}$ are dimensionless variables. In the continuous wavelet literature the scale is denoted by $a$ and the position by $b$ to recall that this transform is based on the affine group $a x+b$. We prefer here to denote the scale $l$, because it corresponds to the length scale at which we analyze $f(\mathbf{x})$, and the position of the analyzing wavelet $\mathbf{x}^{\prime}$, because it indeed corresponds to the actual position in physical space; we must distinguish $\mathbf{x}^{\prime}$ and $\mathbf{x}$, which will be used as an integration variable in (6). The rotation matrix $\Omega$ belongs to the group $S O(n)$ of rotations in $\mathbf{R}^{n}$, and depends on the $n(n-1) / 2$ Euler angles $\boldsymbol{\theta}$. The factor $l^{-n / 2}$ is a normalization which causes all the wavelets to have the same $L^{2}$ norm; 


\section{FARGE}

therefore all wavelets will have the same energy and the wavelet coefficients will correspond to energy densities.

The admissibility condition (1) implies that the Fourier transform of $\psi$ is rapidly decreasing near $k=0$. Therefore the Fourier transform of the family $\psi_{i \times \cdot \theta}$ constitutes a bank of passband filters with constant ratio of width to center frequency:

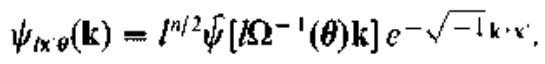

To summarize, the family of analyzing wavelets $\psi_{t^{\prime} \theta}$ may be compared to a mathematical microscope and polarizer, for which $\psi$ characterizes the optics, $I^{-1}$ is the resolution, $\mathbf{x}^{\prime}$ the position, and $\theta$ the polarization angle.

The continuous wavelet transform of a function or a distribution $f(x)$ is the $L^{2}$-inner product between $f$ and the wavelet family $\psi_{t x^{\prime}}$, which gives the wavelet coefficients:

$$
f\left(l, \mathbf{x}^{\prime}, \boldsymbol{\theta}\right)=\left\langle\psi_{t^{\prime} \theta} \mid f\right\rangle=\int_{\mathrm{R}^{n}} f(\mathbf{x}) \psi_{\mathrm{x}^{\prime} \theta}^{*}(\mathbf{x}) d^{n} \mathbf{x},
$$

where $\psi^{*}$ is the complex conjugate of $\psi$, or likewise the inner product between $\hat{f}$ and the filter bank $\hat{\psi}_{\mathbf{x}^{\prime} \hat{\theta}}$, which gives:

$$
f\left(l, \mathbf{x}^{\prime}, \boldsymbol{\theta}\right)=\int_{\mathbf{R}^{n}} f(\mathbf{k}) \psi_{l \mathbf{x}^{*} \theta}^{*}(\mathbf{k}) d^{n} \mathbf{k} .
$$

Figure 2 shows the continuous wavelet transform of some "academic" signals chosen as limiting cases of a hypothetical turbulent signal: a delta function, a period-doubling signal, and a Gaussian white noise signal.

WAVELET SYNTHESIS The admissibility condition (1) implies the existence of a reproducing kernel (which will be defined in Section 3,2). We can therefore recover the signal $f(\mathbf{x})$ from its wavelet coefficients:

$$
f(\mathbf{x})=C_{\psi}^{-1} \int_{0^{+}}^{+\infty} \int_{\mathbb{R}^{n}} \int f\left(l, \mathbf{x}^{\prime}, \boldsymbol{\theta}\right) \psi_{l \mathbf{x}^{\prime} \boldsymbol{\theta}}(\mathbf{x}) \frac{d l d^{n} \mathbf{x}^{\prime}}{l^{s+1}} d \mu(\boldsymbol{\theta}) .
$$

If $\psi$ is complex-valued and $f$ real-valued we should only take the real part of (8).

For one-dimensional or for isotropic wavelets we need not integrate over angles. Otherwise we would have to carry out the following procedure. In order to integrate over the $n(n-1) / 2$ Euler angles $\theta$, we define the integral:

$$
\int d \mu(\theta)=\int_{0}^{2 \pi} \cdots(n-1)+\cdots \int_{0}^{\pi} \cdots(n) \cdots d \mu(\theta) .
$$


A Annual Reviews

www.annualreviews.org/aronline

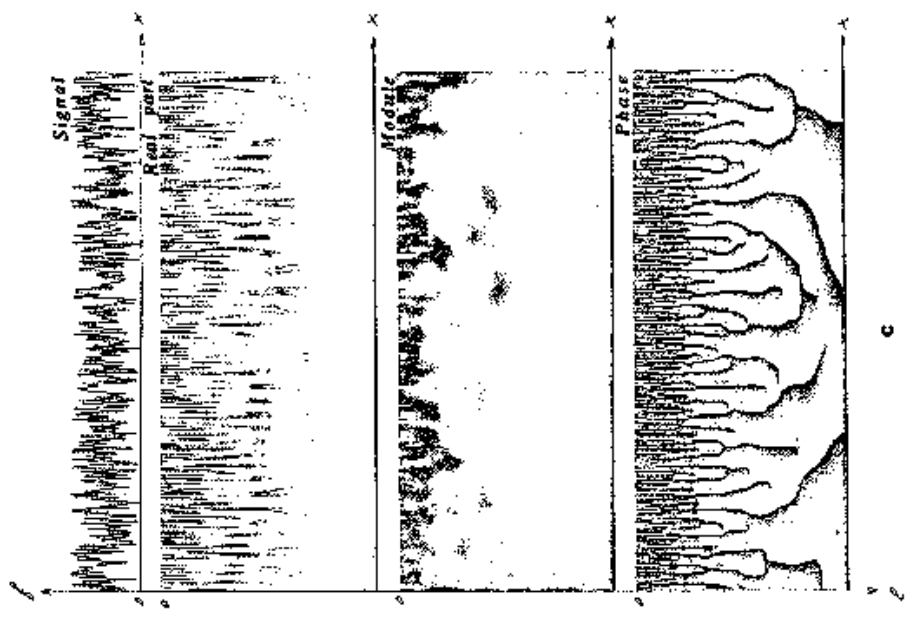

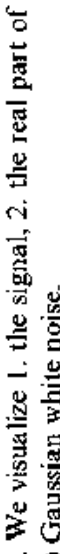

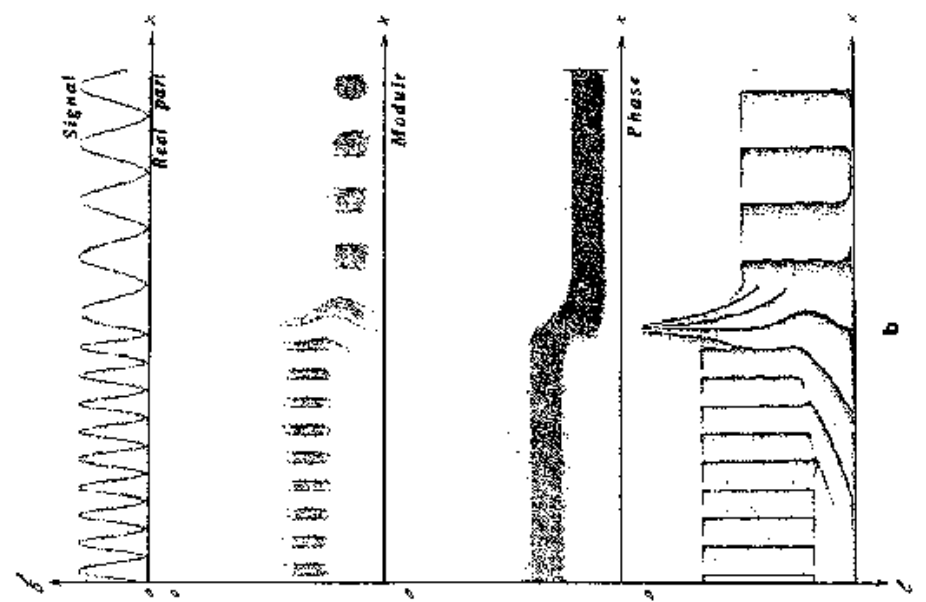

iI

$\rightarrow$ ํํㄴ

동

要

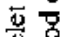

究

3 잉

혼

造 은

解

독

㝵

崽

总

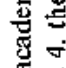

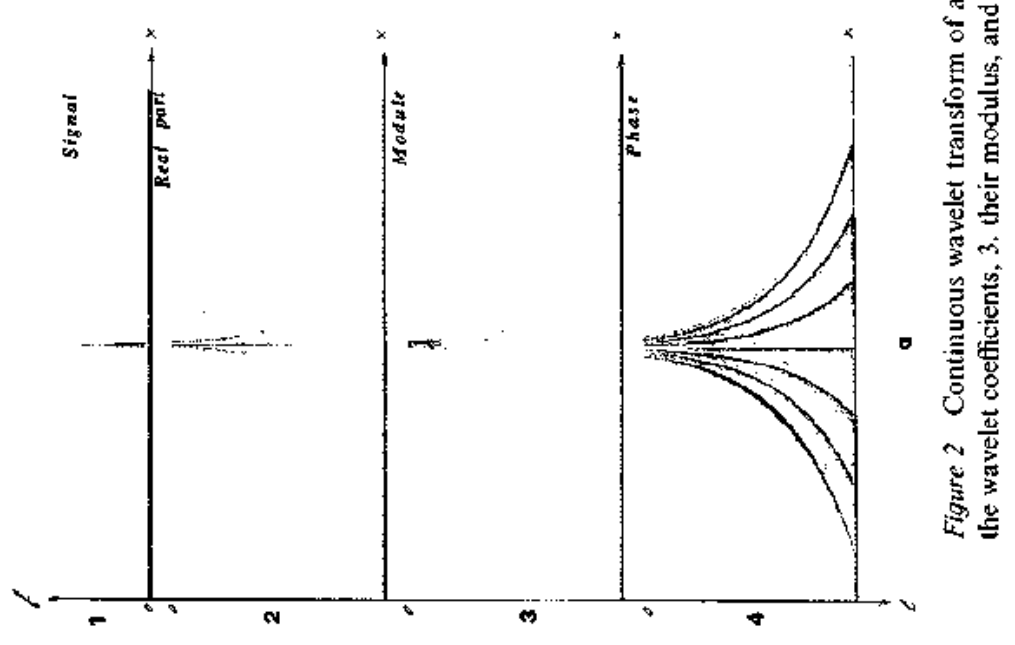




\section{FARGE}

In this integral we use the invariant measure $d \mu(\theta)$ of the rotation group $\mathbf{S O}(n)$ defined as:

$$
d \mu(\theta)=A_{n} \prod_{k=1}^{n-1} \prod_{j=1}^{k} \sin ^{j-1} \theta_{j}^{k} d \theta_{j}^{k}
$$

with

$$
A_{n}=\prod_{k=1}^{n} \frac{\Gamma\left(\frac{k}{2}\right)}{2 \pi^{k / 2}}
$$

and the Euler angles

$$
\begin{cases}0 \leq \theta_{1}^{k}<2 \pi & \text { for } k \in[1, n-1], \\ 0 \leq \theta_{j}^{k}<\pi & \text { for } \quad j \neq 1 \text { and } k \in[1, n-1] .\end{cases}
$$

By analogy with Fourier space, we shall call "wavelet space" the set of functions $f$ that are wavelet transforms of $f$ for a given wavelet $\psi$.

The continuous wavelet transform isometrically transforms a function of $n$ variables into, either an $(n+1)$-dimensional wavelet space if we use an isotropic wavelet, or an $\{n[(n+1) / 2]+1\}$-dimensional wavelet space if we consider a directional wavelet. Therefore the information contained in the wavclet cocfficients is redundant, which is expressed by the reproducing kernel property of the continuous wavelet transform (Section 3.2); consequently there exist many different reconstruction formulas. For instance, it is possible to reconstruct $f(\mathbf{x})$ from its wavelet coefficients using another function, the synthesizing wavelet, different from the analyzing wavelet, which then must verify a modified admissibility condition (Holschneider 1988a, Holschneider \& Tchamitchian 1989). We can even choose a distribution such as the delta function $(\delta)$ to reconstruct the signal. In this case we get the simple reconstruction formula, found empirically by $\mathbf{J}$. Morlet:

$$
f(\mathbf{x})=C_{\delta} \cdot \int_{0^{+}}^{+\infty} \int f(l, \mathbf{x}, \theta) \frac{d l}{l^{1+(n / 2)}} d \mu(\theta)
$$

with

$$
C_{\delta}=(2 \pi)^{n / 2} \int_{\mathbf{R}^{n}} \hat{\psi}(\mathbf{k}) \frac{d^{n} \mathbf{k}}{|\mathbf{k}|^{n}} .
$$

We mention incidentally that we can use a wavelet to synthesize a signal from what are called its Radon transform coefficients, which may be interesting for tomographic applications (Holschneider 1990). 


\subsection{Elementary Properties}

We will now list some of the main properties of the continuous wavelet transform. For the sake of simplicity, from here on we will consider the one-dimensional wavelet transform, the generalization to $n$ dimensions being straightforward, and discard the prime in $x^{\prime}$. We will denote the continuous wavelet transform of a function $f(x)$ by the operator notation $W[f](x)$, and the resulting wavelet coefficients by $f(l, x)$.

LINEARITY The wavelct transform is lincar becausc it is an inner product between the signal $f$ and the wavelet $\psi$. Likewise, the continuous wavelet transform of a vector function is a vector whose components are the continuous wavelet transform of the different components.

COVARIANCE BY TRANSLATION AND DILATION The continuous wavelet transform is covariant under any translation $x_{0}$ :

$$
W[f]\left(x-x_{0}\right)=f\left(l, x-x_{0}\right),
$$

which in particular implies that differentiation commutes with continuous wavelet transform; namely we have

$$
\begin{aligned}
& \frac{\partial}{\partial x} W(f)=W\left(\frac{\partial f}{\partial x}\right), \\
& \nabla[W(\mathbf{f})]=W(\nabla \mathbf{f}), \\
& \nabla \cdot[W(\mathbf{f})]=W(\nabla \cdot \mathbf{f}) .
\end{aligned}
$$

A consequence of the translation covariance is the fact that the frequency of a monochromatic signal can be read off from the phase of the wavelet coefficients (Escudié \& Torrésani 1989, Guillemain 1991, Tchamitchian \& Torrésani 1991, Delprat et al 1991). The number of zeros of the phase on lines with $l=$ constant gives the frequency of the signal (Figure $2 b$ ). This property is independent of the wavelet chosen.

The continuous wavelet transform is also covariant under any dilation by $l_{0}$ :

$$
W\left[f\left(l_{0} x\right)=l_{0}^{-1} \tilde{f}\left(l_{0} l, l_{0} x\right) .\right.
$$

A consequence of the dilation covariance is the fact that the wavelct transform of a power-law function is fully determined by its restriction to any line $l=$ constant. The lines of constant phase point out the possible singularities of the function (Figure $2 a$ ). This property is also independent of the wavelet choice. 
408

DIFFERENTIATION

$$
W\left[\frac{\partial^{m} f}{\partial x^{m}}\right]=(-1)^{m} \int_{-\infty}^{+\infty} f(x) \frac{\partial^{m}}{\partial x^{m}}\left[\psi_{x i}^{*}(x)\right] d x .
$$

ENERGY CONSER VATION

$$
\int_{-\infty}^{+\infty}|f(x)|^{2} d x=C_{\psi}^{-1} \int_{0^{+}}^{+\infty} \int_{-\infty}^{+\infty}|f(l, x)| \cdot\left|f^{*}(l, x)\right| \frac{d l d x}{l^{2}}
$$

The wavelet transform conserves energy not only globally but also locally if one considers all coefficients inside the "influence cone" which consists of the spatial support of all dilated wavelets. The above relation corresponds to energy conservation, which is a generalization of the Plancherel identity to the continuous wavelet transform. It implies that there is no loss of information in transforming the signal into its wavelet coefficients.

The total energy can also be split among the different scales $l$ :

$$
E(l)=C_{\psi}^{-1} \int_{-\infty}^{+\infty}|\tilde{f}(l, x)|^{2} \frac{d x}{l^{2}}
$$

SPACE-SCALE LOCALITY The space-scale locality of the analyzing wavelet $\psi$ leads to the conservation of locality in the wavelet coefficient space, in contrast to the Fourier transform which loses the locality present in the signal. For instance, if $\psi$ is well-localized in the space interval $\sigma_{x}$ for $l=1$, then the wavelet coefficients corresponding to the position $x_{0}$ will all be contained in the influence cone defined by $x \in\left[x_{0}-\left(l \cdot \sigma_{x}\right) / 2, x_{0}+\left(l \cdot \sigma_{x}\right) / 2\right]$. This cone corresponds to the spatial support of all dilated wavelets at the point $x_{0}$. Likewise, if $\hat{\psi}$ is well-localized in the Fourier interval $\Delta k$ around $k_{\psi}$ for $l=1$, then the wavelet coefficients corresponding to the Fourier frequency $k_{0}$ in the signal will only be contained in the subband defined by $l \in\left[k_{\psi}\left(k_{0}+(\Delta k / 2 l)\right)^{-1}, k_{\psi}\left(k_{0}-(\Delta k / 2 l)\right)^{-1}\right]$.

LOCAL REGULARITY ANALYSIS One of the most interesting properties of the continuous wavelet transform, implied by its dilation covariance, is the possibility it offers to measure the local regularity of a function and therefore to characterize the functional space to which it belongs ( $\mathrm{Hol}-$ schneider 1988a,b; Jaffard 1989a,b, 1991c; Holschneider \& Tchamitchian 1989, 1990; Tchamitchian 1989c). For instance if $f \in C^{m}\left(x_{0}\right)$, i.e. if $f$ is continuously differentiable in $x_{0}$ up to order $m$, then

$$
\widetilde{f}\left(l, x_{0}\right) \lesssim l^{m+1} l^{1 / 2} \text { for } l \rightarrow 0 \text {. }
$$

The factor $l^{1 / 2}$ comes from the fact that, due to the scale invariance (14), 
if we want to study the scaling of a function we must take the wavelet coeflicients in the $L^{1}$ norm, instead of $L^{2}$. The wavelet coefficients written in the $L^{1}$ norm are related to the wavelet coefficients written in the $L^{2}$ norm by the simple expression:

$$
f_{L^{1}}=t^{-1 / 2} f_{L^{2}}
$$

If $f$ belongs to $\Lambda^{\alpha}\left(x_{0}\right)$, the Hölder space of functions of exponent $\alpha$, i.e. if $f$ is continuous, not necessarily differentiable in $x_{0}$ but such that

$$
\left|f\left(x+x_{0}\right)-f(x)\right|=C\left|x_{0}\right|^{\alpha} \quad \text { with } \alpha<1 \text { and constant } C>0,
$$

then

$$
f\left(l, x_{0}\right) \sim C e^{\sqrt{-1} \Phi l^{\alpha} l^{1 / 2}} \text { for } l \rightarrow 0,
$$

where $\Phi$ is the phase of the wavelet coefficients.

The transformed function $f$ is regular even if $f$ is not. The information about any possible singularities present in the signal, their position $x_{0}$, their strength $C$, and their scaling exponent $\alpha$, is given by the asymptotic behavior of $\tilde{f}\left(l, x_{0}\right)$, written in norm $L^{\prime}(19)$, for $l$ tending to zero. If the $L^{\prime}$-norm wavelet coefficients diverge in the small scales at the point $x_{0}$, then $f$ is singular al $x_{0}$ and the slope of $\log \left|f\left(l, x_{0}\right)\right|$ versus $\log l$ will give the exponent of the singularity (20). It is also very easy to localize the possible singularities of $f$ by looking at the phase of its wavelet coefficients: The lines of constant phase converge on the singularities (Figure $2 a$ ). If, on the contrary, the modulus of the wavelet coeflicient becomes zero in the very small scales around $x_{0}$, then the function $f$ is regular at $x_{0}$. This result is in fact the converse of (18), but its mathematical justification requires more global assumptions on the analyzed function, namely some decay or its wavelel coefficients in the vicinity of $x_{0}$ (Jaffard 1989a). Therefore, in practice, to locally analyze a singularity at $x_{0}$ we should first verify that at small scales the wavelet coefficients around $x_{0}$ are not larger than those at $x_{0}$. Then we should consider not only the coefficients $f\left(l, x_{0}\right)$, but at least all coefficients betonging to the influence cone pointing towards $x_{0}$. Those properties are independent of the choice of the wavelet and they are particularly useful for characterizing fractals and multifractals (Holschneider 1988b; Arnéodo et al 1989; Ghez \& Vaienti 1989; Argoul et al 1988, 1990; Falconer 1991; Freysz et al 1990).

REPRODUCING KERNEL Using the wavelet $\psi$, we can decompose any function or distribution $f$ into its wavelet coefficients. In the case of the continuous wavelet transform these coefficients form an over-complete basis. This implies a correlation between the wavelet coefficients which, in turn, corresponds to the existence of a reproducing kernel $K_{\psi}$ associated to the wavelet $\psi$ defined by 


\section{FARGE}

$$
K_{\psi}\left(l_{1}, l_{2}, x_{1}^{\prime}, x_{2}^{\prime}\right)=C_{\psi}^{-1} \int_{-\infty}^{+\infty} \psi_{l_{1} x^{\prime}}, \psi_{l_{2} x_{2}^{\prime}}^{*} d x .
$$

$K_{\psi}$ characterizes the correlation of the continuous wavelet transform between two different points of the continuous half-plane $(l, x)$. Its structure depends on the choice of the wavelet; indeed the reproducing kernel measures the space and scale selectivity of each wavelet $\psi_{i x}$ and will therefore be very useful in helping to choose the wavelet most appropriate to a given problem.

Reciprocally, an arbitrary function $f$ of the half-plane $(l, x) \in\left(\mathbf{R}^{+} \times \mathbf{R}\right)$ is not in general the wavelet transform of some function of $\mathbf{R}$ with respect to a given wavelet $\psi$. This will hold only if $f$ is square integrable and satisfies the reproducing kernel equation (Grossmann et al 1989, Grossmann \& Kronland-Martinet 1988):

$$
f\left(l_{1} x^{\prime}\right)=\int_{u^{+}}^{+\infty} \int_{-\infty}^{+\infty} K_{\psi}\left(l_{1}, l_{2}, x_{1}^{\prime}, x_{2}^{\prime}\right) f\left(l_{2}, x_{2}^{\prime}\right) \frac{d l_{2} d x_{2}^{\prime}}{l_{2}^{2}} .
$$

This condition should be checked if we want to partially resynthesize a signal from a filtered subset of its wavelet space. An important consequence of the reproducing kernel property (22) is the fact that the continuous wavelet transform of a random signal (Figure $2 c$ ) shows some correlations that are obviously not in the signal, but in the wavelet transform itself. The size of the correlated regions is given by the reproducing kernel and decreases with the scale. This is one of the most common pitfalls of the continuous wavelet transform and one should be particularly aware of it when studying turbulent signals. In the case of discrete orthogonal wavelet transform (Section 4.2) this problem no longer exists, since by definition of orthogonality all wavelet coefficients are uncorrelated.

\subsection{Implementation}

WAVELET CHOICE As we have seen, the wavelet transform is an inner product between an analyzing wavelet at a given scale $l$ and the signal to be analyzed; therefore the wavelet coefficients combine information about both the signal and the wavelet. The choice of the transform, orthogonal or not, and of the appropriate wavelet is thus an important issue which depends on the kind of information we want to extract from the signal. For analyzing purposes the continuous wavelet transform is better suited because its redundancy allows good legibility of the signal's information content. For compression or modeling purposes, the orthogonal wavelet transform (Section 4.2) or the newly developed wavelet packet technique (Section 4.3) are preferable because they decompose the signal into a 
minimal number of independent coefficients. Then for choosing the appropriate wavelet, we must look at its reproducing kernel (21), which characterizes its space, scale, and angular selectivity.

EXAMPLES OF COMPLEX-VALUED WAVELETS Let us consider the analysis of a real-valued signal such as those encountered in fluid mechanics. In this case, we usually choose a continuous wavelet transform with a progressive complex-valued wavelet, because the quadrature $\pi / 2$ phase shift between its real and its imaginary parts allows us to eliminate the wavelet's oscillations when visualizing the wavelet coefficient modulus (Figure 2). From the resulting complex-valued wavelet coefficients we can thus separate the $L^{2}$ modulus, which gives the energy density, and the phase, which detects singularities and measures instantaneous frequencies (Escudié \& Torrésani 1989, Tchamitchian \& Torrésani 1991, Guillemain 1991, Delprat et al 1991). As already stated, the lines of constant phase converge on singularities and the number of zero-crossings on lines $l=$ constant, if the latter are parallel, is related to the signal frequency. The phase behavior is independent of the choice of wavelet.

The most commonly used complex-valued wavelet is the Morlet wavelet (Figure 3a).

$$
\psi(x)=e^{\sqrt{-1} k_{\psi} \cdot x} e^{-\left(|x|^{2} / 2\right\}},
$$

which is a plane wave of wavevector $k_{\psi}$, modulated by a Gaussian envelope of unit width. Incidentally, the Morlet wavelet is only marginally admissible, because it is of zero average only if some very small correction terms are added. In practice, if we take $\left|k_{\psi}\right|=6$, the correction terms become unnecessary because they are of the same order as typical computer roundoff errors. Another way to ensure admissibility is to impose $\hat{\psi}(0)=0$. In Fourier space, the Morlet wavelet is given by:

$$
\begin{cases}\hat{\psi}(k)=(2 \pi)^{-1 / 2} e^{-\left(k-k_{\psi}\right)^{2} / 2} & \text { for } k>0 \\ \sqrt{k}(k)=0 & \text { for } k \leq 0\end{cases}
$$

A very interesting property of the Morlet wavelet in its generalization to $n$ dimensions is its angular selectivity, which gets better and better as $\left|\mathbf{k}_{\downarrow}\right|$ increases, but with a concomitant reduction in its spatial selectivity. In order to have both, angular and spatial selectivity Antoine and coworkers have proposed to elongate the Morlet wavelet, while keeping $\left|\mathbf{k}_{\psi}\right|$ small enough to ensure a good space locatization (Antoine et al 1990. Antoine et al 1991).

Another complex-valued wavelet, mostly used in quantum mechanics 

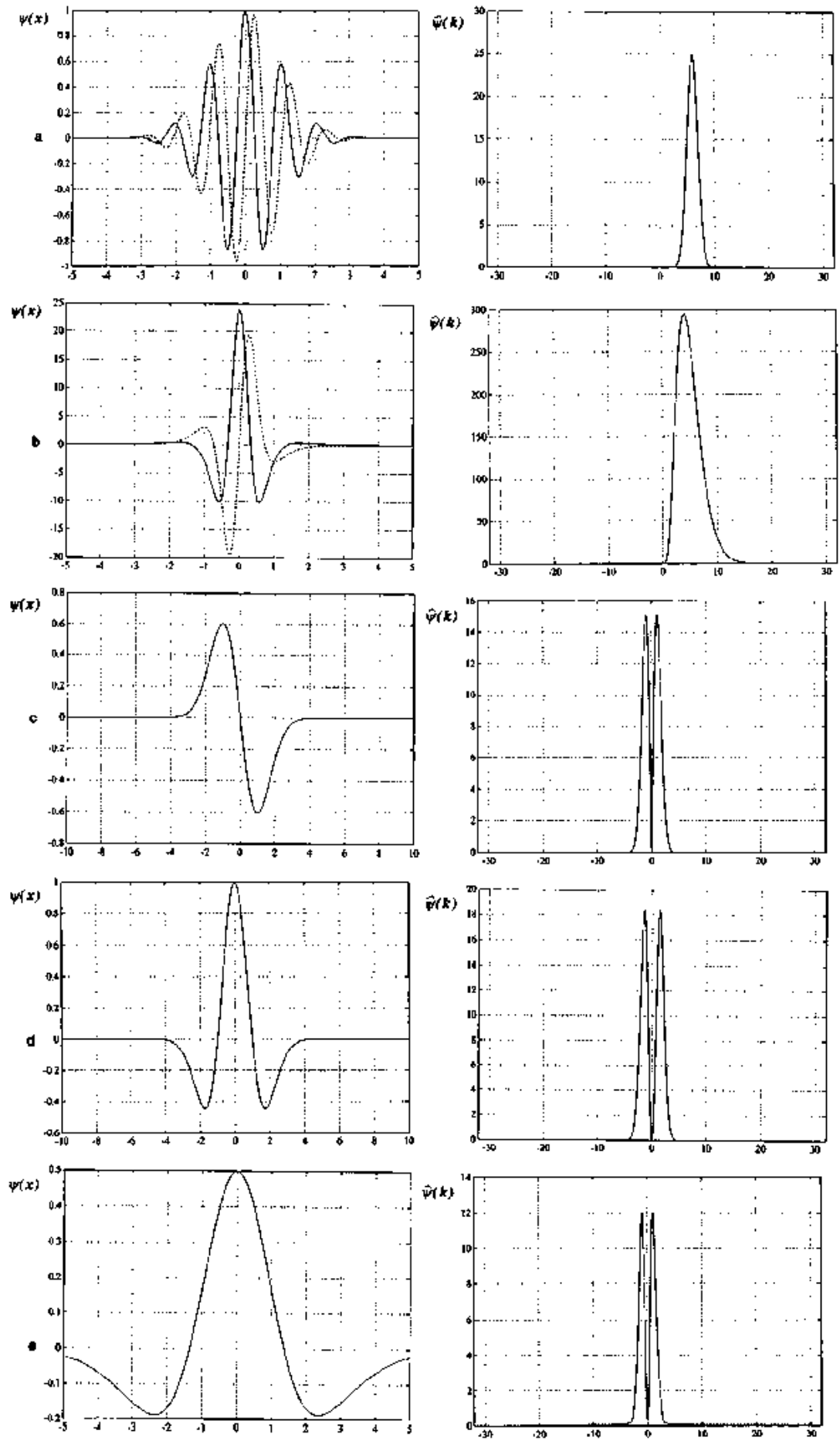
(Paul 1984, 1985a,b, 1986, 1989), is the Paul wavelet which is analytic (Figure 3b):

$$
\psi_{m}=\Gamma(m+1) \frac{(\sqrt{-1})^{m}}{(1-\sqrt{-1} x)^{1+m}},
$$

or

$$
\begin{cases}\hat{\psi}_{m}(k)=k^{m} e^{-k} & \text { for } k>0, \\ \hat{\psi}_{m}(k)=0 & \text { for } k \leq 0 .\end{cases}
$$

The higher the order $m$, the better the cancellations (vanishing moments) of the wavelet. The complex-valued wavelets are called progressive wavelets if their Fourier coefficients are zero for negative wavenumbers. They are well adapted to analyze causal signals, i.e. signals for which there is some action of causality. This is because progressive wavelets preserve the direction of time and do nol create parasitic interference between the past and future. Progressive wavelets are used in particular to analyze musical sounds (Kronland-Martinet et al 1987, Kronland-Martinet 1988).

EXAMPLES OF REAL-VALUED WAVELETS Some commonly used real-valued wavelets are the $m$ th derivatives of the Gaussian (Figures $3 c$ and $3 d$ ):

$$
\psi_{m}(x)=(-1)^{m} \frac{d^{m}}{d x^{m}}\left(e^{-|x|^{2 / 2}}\right)
$$

or

$$
\hat{\psi}_{t n}(k)=m(\sqrt{-1} k)^{m} e^{-\left.|k|\right|^{j} / 2} .
$$

The higher order derivatives imply more cancellations (vanishing moments) of the wavelet. Among these derivatives of the Gaussian, the most widely used is the Marr wavelet, also called the "Mexican hat" (Figure $3 d)$, which is the Laplacian of a Gaussian $(m=2)$. The Marr wavelet in its generalization to $n$ dimensions is isotropic and therefore cannot discriminate different directions in the signal. Jaffard (1991b) has proposed to use, instead of a Laplacian of a Gaussian, a Gaussian differentiated in only one direction, which will then be nonisotropic with a good

Figure 3 Examples of wavelets $\psi$ commonly used for the continuous wavelet transform (continuous line for the real part and broken line for the imaginary part). Wc visualize $\psi(x)$ (lefi) and $\hat{\psi}(k)$ (right). (a) Morlet wavelet for $k_{\psi}=6,(b)$ Paul's wavelet for $m=4$, (c) First derivative of a Gaussian, (d) Marr wavelet (second derivative of a Gaussian), (e) DOG (Difference of two Gaussians). 


\section{FARGE}

angular selectivity. Antoine and co-workers have proposed to elongate it to recover some angular selectivity (Antoine et al 1990, Antoine et al 1991).

Another possible real-valued wavelet is the D.O.G., Difference of Gaussians (Figure 3e), which is a discrete approximation to the Laplacian of a Gaussian:

$$
\psi(x)=e^{-|x|^{2} / 2}-\frac{1}{2} e^{-|x|^{2} / 8},
$$

or

$$
\hat{\psi}(k)=(2 \pi)^{-1 / 2}\left[e^{-|k|^{2} / 2}-e^{-2|k|^{2}}\right] .
$$

Dallard and Spedding have proposed an isotropic version of the twodimensional Morlet wavelet, called the Halo wavelet (Dallard \& Spedding 1990), which is then real-valued but does not have (as the Morlet wavelet) zero-mean value unless one enforces it:

$$
\begin{aligned}
& \hat{\psi}(\mathbf{k})=e^{-\left(|\mathbf{x}|-\left|\mathbf{k}_{\psi}\right|\right)^{2} / 2} \\
& \hat{\psi}(0)=0 .
\end{aligned}
$$

From a real-valued wavelet, which is self-conjugated, i.e. such that $\hat{\psi}(k)=\hat{\psi}^{*}(-k)$, we can always construct a progressive complex-valued wavelet. For this cancels its Fourier coefficients with negative wavenumbers. The procedure is straightforward in one dimension, but it becomes less obvious in the $n$-dimensional case, where the definition of negative wavenumbers is purely conventional. This problem has been recently addressed by Dallard and Spedding, who proposed, using such a method, the construction of a complex-valued isotropic Morlet wavelet in two dimensions, named the Arc wavelet (Dallard \& Spedding 1990). Unfortunately the Arc wavelet presents several problems: Its imaginary part is neither isotropic nor well-localized in physical space. In fact it is probably impossible to construct an isotropic complex-valued wavelet. In practice, if one wants to combine both isotropy and complex-value, Farge and coworkers have proposed to compule a Morlet wavelet transform for $n$ angles, $n$ large enough relative to the angular selectivity of the reproducing kernel. They then integrate the modulus of the wavelet coefficients, but not the coefficients themselves, over all $n$ angles (Farge et al 1990).

WAVELET COEFFICIENT REPRESENTATIONS After choosing the wavelet, we also have to choose the most appropriate graphical representation of the wavelet coefficients. The most commonly used representation is to compute the wavelet coefficients in the $L^{2}$-norm and visualize them, with a linear scale for $x$ and a logarithmic scalc for $l$, using the full color range-for instance between 0 and 255 color levels when data are coded on 1 byte 
at each scale. This normalization of the wavelet coefficients, performed scale by scale, enhances the small-scale coefficients, but we cannot then compare the coefficient amplitudes between different scales. Therefore we should not renormalize if we want to compare the energy density at different scales.

If one is not interested in the energy density but rather in the scaling properties of the wavelet coefficients, another solution is to compute them with the $L^{\prime}$-norm (19) instead of a $L^{2}$-norm. It is then no longer necessary to renormalize the coefficients at each scale because all coefficients, whatever the scale, will now have a similar range of valucs.

Some authors use a linear scale for $l$, but this is not recommended because the small-scale behavior - which is in general the most interesting to study - is then completely flattened. In any case, a logarithmic representation of the scale is natural for wavelets, because it corresponds to the multiplicative nature of the dilation parameter. For instance, in the case of orthogonal wavelets (Section 4.2), we should take the base 2 logarithm, since the dilation parameter is usually a multiple of 2 , and we should therefore represent the wavelet coefficients octave by octave.

ALGORITHMS The root of the continuous wavelet analysis algorithm is a set of convolution products between the signal $f$ and all dilated and rotated wavelets $\psi_{10}$ defined in Equation (4). Therefore the first step of the algorithm will be to generate the family of all dilated and rotated wavelets $\psi_{1 \theta}$ defined in Equation (4). We then perform the convolution product, either in physical space by integrating (6) over all discretized positions $x=i \cdot \Delta x$, or in Fourier space using a FFT (Fast Fourier Transform) and multiplying $\hat{\psi}_{i \theta}$ defined in (5) and $\hat{f}$ before transforming the result back to physical space to obtain $\tilde{f}_{t x p}$.

In all cases, we must check that the wavelet sampling remains sufficient to compute the smallest scale $l_{\min }$, in order to minimize numerical errors and avoid aliasing. Incidentally one should notice that the computation of the large-scale wavelet coefficients requires a wavelet sampling as good as the signal sampling. We should also ensure that, when computing the large scales, we still have enough of the signal on the left and on the right while translating $\psi_{10}$. If such is not the case, we should extend the signal by keeping its left $f\left(x_{\min }\right)$ and right $f\left(x_{\max }\right)$ values constant, or make it decay smoothly to 7ero; in this case the wavelet coefficients inside both influence cones (Section 3.2), associated with $x_{\min }$ and $x_{\max }$, respectively, would be meaningless. To avoid such side effects, the best solution would be to make the signal periodic, if it is not already so.

The best way to test the wavelet analysis algorithm, is to compute the wavelet transform of a Dirac function, which should give the analyzing 


\section{FARGE}

wavelet at each scale (Figure $2 a$ ). To check if the spatial and angular samplings are sufficiently dense, we should compute the reproducing kerncl (Section 3.2), i,e, the wavelet transform of the analyzing wavelets themselves, considering only the wavelet at scale $l_{\min }$ and angle $\theta_{0}$; then we should plot, for any point $x_{0}$, the wavelet coefficients in $l, \theta$ polar coordinates. If we do not see any spurious side effects, the sampling is therefore sufficient.

The simplest algorithm for continuous wavelet synthesis is to compute the Morlet formula (11), which uscs a Dirac function as a synthesizing wavelet, because this formula minimizes the number of integrations. Due to numerical errors in the wavelet analysis algorithm, the reconstruction of the signal will not be exact. If we want to ensure an exact reconstruction, we should have computed the wavelet analysis in physical space by integrating (6) using an interpolation basis associated with the sampled signal $f$, instead of the sampled signal itself.

For a one-dimensional signal sampled over $I$ points which has a very large number of scalcs $J$ (in audioacoustics for instance the scales range typically from 1 to $2^{10}$, the computing time may soon become prohibitive, because it would vary as $J \cdot I^{2}$, or $J \cdot I \cdot \log _{2} I$ if we use the FFT. In this case there exists a fast algorithm, called "algorithme à trous" (Holschneider et al 1988, 1989; Dutilleux 1989), which, at each scale, keeps constant the number of sampled points for the wavelet and thus avoids the oversampling of the wavelet which was necessary to compute the large-scale coefficients; it computes only $I /\left(\lambda^{J}\right)$ points for the signal, $\lambda$ being the ratio between two successive scales $\lambda=l_{j+1} / l_{j}$. The operation count is then proportional to $J \cdot I \cdot \log _{\lambda} I$, without requiring signal periodicity as docs the FFT algorithm. The wavelet coefficients are only computed on the incomplete grid ("grille à trous") of sizc $J \cdot \log _{\lambda} I$ and not on the complete grid of size $J \cdot I$; we must then usc an appropriate interpolation (Section $4.1)$ if we want to compute all the cocfficients of the complete grid. For $\lambda=2$, namely if the scales vary octave by octave, the "algorithme à trous" is very similar to the Mallat algorithm (Section 4.2) developed for orthogonal wavelets, but without requiring orthogonal wavelets.

\section{THE DISCRETE WAVELET TRANSFORM}

\subsection{Wavelet Frames}

DEFINITION In a sense, the analysis (6) and synthesis (8) formulas work as if the functions $\psi_{k}, l \in \mathbf{R}^{+}$and $x \in \mathbf{R}$, constituted an orthogonal complete set of $L^{2}(\mathbf{R})$ : The coefficients of the decomposition of $f(x)$ in this basis are given by (6) and the reconstruction of $f(x)$ from these coefficients is given by (8). In fact, for the continuous case the sct of functions $\psi_{t x}$ is highly redundant. Is it possible to select a subset $\mathbf{F}$, called a "wavelet frame," such 
that the $\psi_{j l} \in \mathbf{F}$ would constitute a complete set that is almost orthogonal for $L^{2}(\mathbf{R})$ ? The answer is yes, but only approximately (Daubechies et al 1986, Daubechies 1990). We proceed with the following discretization of the half-plane $l, x: l$ is logarithmically sampled at intervals of $(\Delta \log l)^{-j}$, $j \in \mathbf{Z}$, and $x$ is linearly sampled, with an increment that depends on the scale, at intervals of $i \cdot \Delta x \cdot(\Delta \log l)^{-j}, i \in \mathbf{Z}$. Thus the measure $d l \cdot d x / l^{2}$ in Equation (8) becomes $(\Delta \log l)^{-2 j}$. The corresponding discrete analysis formula is

$$
\tilde{f}_{j i}=\left\langle\psi_{j i} \mid f\right\rangle=\int_{-\alpha i}^{+\infty} f(x) \psi_{j i} \cdot d x
$$

with

$$
\psi_{j i}(x)=(\Delta \log l)^{j / 2} \psi\left[(\Delta \log l)^{j} x-i \Delta x\right],
$$

for $j \in \mathbf{Z}, i \in \mathbf{Z}$, and the discrete reconstruction formula is

$$
f(x)=C \sum_{j=-\infty}^{+\infty} \sum_{j=-\infty}^{+\infty} \tilde{f}_{j} \psi_{j i}+R,
$$

where $C$ is a constant and $R$ is a residual which is zero only if the frame is orthogonal. $\Delta \log l$ can in general be made small enough so that $R$ can be neglected, and we then have a quasi-orthogonal frame.

QUASI-ORTHOGONALITY The reconstruction formula is only an approximation from which, by iteration, one can obtain an exact reconstruction of $f(x)$ when $C \sim 1$ and $R \sim 0$ (Daubechies et al 1986, Daubechies 1987). This optimal frame is then quasi-orthogonal and complete (tight frame). For instance let us consider the Marr wavelet, $\psi(x)=\left(1-x^{2}\right) e^{-x^{2} / 2}$. Its optimal frame, namely that which minimizes $R$ in (30), corresponds to $\Delta \log l=2^{1 / 2}$ and $\Delta x=1 / 2$ (Daubechies 1989). With this frame the error in the energy for the reconstruction of $f(x)$ is less than $2 \times 10^{-5}$, which is sufficient for most applications. The discretization $\Delta \log l=2$ and $\Delta x=1$ corresponds to the dyadic grid (Figure $4 a$ ), for which it is also possible to construct some exactly orthogonal wavelet bases (Section 4.2). For the Morlet wavelet (23) its optimal frame corresponds to the dyadic grid only when $\left|k_{\psi}\right|=3$.

INTERPOLATION With the discrete wavelet transform we have lost the covariance by dilation and translation of the continuous wavelet transform and the redundancy of the wavelet coefficients, both properties which can be very useful for signal analysis and signal processing. It is actually possible, under some additional hypotheses, to recompute the whole set of the continuous wavelet coefficients from a discrete subset of the wavelet coefficients by using an appropriate interpolation (Grossmann \& Morlet 1984, Grossmann et al 1989) based on the reproducing kernel property 


\section{FARGE}
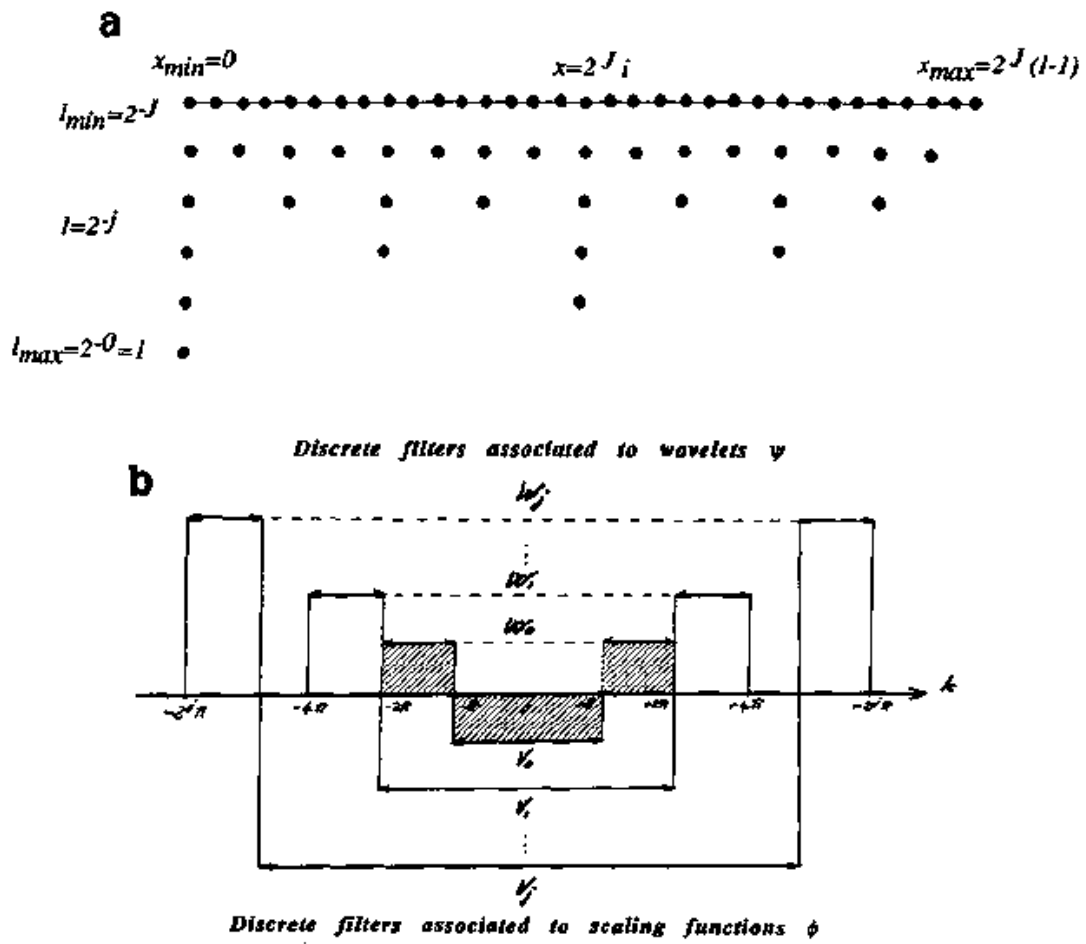

C

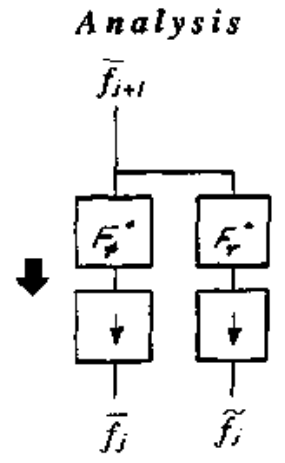
F] Convotution by the duat of the distrese fitier associased to scaliag functions
Deconvolurion by the discrett filser associated to scoling functions
Canvolurion by the duat of the aiscrest fitiser assoctated io waveiers $\psi$
Deconvolution by the discrete filter associared to wovelets $\$$
Oversampling by putting one zero denueen each sample Undersampling by raking one sampie every mo samples
-2 Matriplization by 2

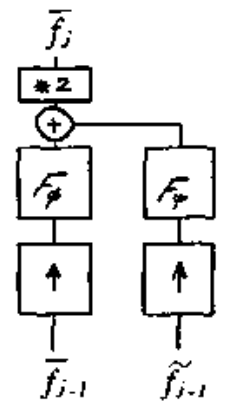

Figure 4 Multiresolution analysis. (a) The dyadic grid, (b) Illustration of the multiresolution principle in Fourier space, $(c)$ Mallat algorithm. 
(Section 3.2) of the continuous wavelet transform. With the frames and the interpolation formula, we now have a complete methodology for extracting the quasi-orthogonal discrete wavelet coefficients from the continuous wavelet coefficients using the wavelet frames and vice-versa to recover the continuous wavelet coefficients by interpolating from the discrete wavelet coefficients. This two-way approach allows us to combine the redundancy and the geometrical properties of the continuous wavelet transform with the economy of the discrete orthogonal wavelet transform. Therefore in practice the distinction between the continuous wavelet transform and the orthogonal wavelet transform is often not so important.

\subsection{Orthogonal Wavelets}

DEFINITION As we have seen, the frames $F$ are quasi-orthogonal complete sets of $L^{2}(\mathbf{R})$. But there also exist some special wavelets $\psi$ such that

$$
\psi_{j i}(x)=2^{j / 2} \psi\left(2^{j} x-i\right), \quad \text { with } j \in \mathbf{Z}, i \in \mathbf{Z},
$$

constitutes a genuine orthogonal basis of $L^{2}(\mathbf{R})$; namely the functions $\psi(x)$ are orthogonal to their translates by discrete steps $x=2^{-i} \cdot i$ and their dilation by $l=2^{-j}$, which corresponds to the dyadic grid (Figure $4 a$ ), and the family (31) is complete in $L^{2}$.

Using an orthogonal wavelet basis $\left\{\psi_{j i}\right\}$, we can decompose any function or distribution $f(x)$, decaying sufficiently fast at infinity, such that

$$
f(x)=\sum_{j=-\infty}^{+\infty} \sum_{i=-\infty}^{+\infty} \tilde{f}_{j i} \psi_{j i}(x)
$$

with

$$
\bar{f}_{j i}=\left\langle\psi_{j i} \mid f\right\rangle=\int_{-\infty}^{+\infty} f(x) \psi\left(x-2^{-y} i\right) d x
$$

Orthogonality implies that the total energy is conserved:

$$
\int_{-\infty}^{+\infty}|f(x)|^{2} d x=\sum_{j=-\infty}^{+\infty} \sum_{i=-\infty}^{+\infty}\left|f_{j i}\right|^{2} .
$$

Contrary to the continuous wavelet transform (Section 3.2) the orthogonal wavelet transform is not covariant by translation and dilation, except by discrete translations $2^{-j} i$ and discrete dilations $2^{-j}$. To recover in practice the translational covariance Mallat and Zhong considered the zero-crossings, or the local extrema, of the orthogonal wavelet coefficients, instead of the coefficients themselves (Mallat \& Zhong 1990, 1991). They have also shown that it is possible to have a unique and complete reconstruction of the signal from the local extrema alone, due to the reproducing kernel 
property (Section 3.2) of the wavelet transform; however this assertion is not true for all functions as Meyer (personal communication) has recently proved.

MULTIRESOLUTION ANALYSIS If we want to generalize the wavelet transform to decompose any function or distribution $f(x)$ whatever its decay at infinity, we must use, in addition to the analyzing wavelet $\psi$, another function $\phi$, called the "wavelets's father" or "scaling function," such that

$$
\int_{-\infty}^{+\infty} \phi(x) d x=1,
$$

where

$$
\phi_{j i}=2^{j / 2} \phi\left(2^{j} x-i\right) \text { is orthogonal to } \psi_{j i} \text { for } j^{f}>j \text { and } \forall i^{t}, i \text {. }
$$

It has been proven (Mallat 1989b) that $\phi_{j k}$ is orthogonal to all its discrete translates.

Consequently the decomposition of $f(x)$ becomes:

$$
f(x)=\sum_{i=-\infty}^{+\infty}\left\langle\phi_{0 i} \mid f\right\rangle \phi_{0 i}(x)+\sum_{i=0}^{+\infty} \sum_{i-\infty}^{+\infty}\left\langle\psi_{j i} \mid f\right\rangle \psi_{j i}(x) .
$$

The first sum is a smooth approximation of $f(x)$ at the largest scale $l_{\text {max }}=2^{0}=1$, while the second sum corresponds to the addition of details of scale $l=2^{-j}, j \in[0,+\infty]$. The function $\hat{\phi}_{0}$ is a low-pass filter, while $\hat{\psi}_{i}$ constitutes a set of orthogonal higher and higher pass filters. This approach, also called multiresolution analysis, is appropriate for analyzing functions which are not in $L^{2}(\mathbf{R})$. Take for example $f(x) \equiv 1$, which is not square integrable (Meyer 1990a). We then have $\left\langle\phi_{0 t} \mid f\right\rangle=1$ and $\left\langle\psi_{j i} \mid f\right\rangle=0$, from which we reconstruct $f(x)=1$; this reconstruction would have been wrong if we had only used the wavelet $\psi$ without the smoothing function $\phi$, such as in Equation (32).

The elegance of the multiresolution analysis comes from the fact that the scaling functions $\phi_{j i}$ generate a set of nested subspaces $V_{0} \subset V_{1} \subset \ldots$ $V_{j} \subset V_{j+1} \ldots$ while the associated wavelets $\psi_{j i}$ constitute their orthogonal complementary subspaces $W_{0}, W_{1} \ldots W_{j}, W_{j+1} \ldots$ such that $V_{j+1}=V_{j} \oplus W_{j}$ (Figure 4b). The inclusion $V_{j} \subset V_{j+1}$ corresponds to a mesh refinement by a factor of 2 :

$$
f(x) \in V_{j} \Leftrightarrow f(2 x) \in V_{j 1} \text {. }
$$

This indicates that the approximation of $f(x)$ at scale $2^{-(j+1)}$ is

$$
\overline{f_{j+1}}(x)=\sum_{i=-\infty}^{+\infty}\left\langle\phi_{(i+1) i} \mid f\right\rangle \phi_{j+1, i}
$$


and contains all the necessary information to compute the same signal at the larger scale $2^{-f}$. When computing an approximation of $f(x)$ at scale $2^{-j}$, some information $f$ is lost, but as the scale decreases to $2^{-\infty}=0$ the approximated signal converges to $f(x)$. Conversely, as the scale increases to $l=\infty$ the approximated signal contains less and less information and converges to zero.

$V_{j}$ is the set of all possible approximations at scale $l=2^{-j}$ of functions in $L^{2}(\mathbf{R})$. Among all approximated functions at scale $l=2^{-j}, \bar{f}(x)$ is the function that is the closest in $L^{2}$-norm to $f(x)$. Therefore the wavelet decomposition is an orthogonal projection on the vector space $V_{j}$

All the smooth approximations $\vec{f}_{j}(x)$ at scale $j$ of $f(x)$ belong to $V_{j}$, while all the additional details $f_{j}(x)$ necessary at scale $j$ to exactly recover $f_{j+1}(x)$ at scale $j+1$ belongs to $W_{j}$

$$
\bar{f}_{j+1}(x)=\bar{f}_{j}(x)+\tilde{f}_{j}(x)
$$

Recursively we obtain the reconstruction formula

$$
f(x)=f_{0}(x)+\sum_{j=0}^{+\infty} f_{j}(x)
$$

which corresponds to the decomposition

$$
L^{2}(\mathbf{R})=V_{0} \underset{j \geq 0}{\oplus} W_{j}
$$

MAI.RAT ALGORITHM An additional simplification introduced by Mallat (Mallat 1989a-d) allows the computation of the scaling function $\psi$ from a discrete filter $F_{\phi}$, similar to the Quadrature Mirror Filters used in signal coding (Esteban \& Galand 1977, Rioul 1991). This filter $F_{\phi}$ comes from the space inclusion $V_{0} \subset V_{1}$ which implies

$$
\hat{\phi}(k)=\bar{F}_{\phi}\left(\frac{k}{2}\right) \hat{\phi}\left(\frac{k}{2}\right) \text { with } \hat{F}_{\phi}(k)=2^{-1 / 2} \sum_{i} F_{\phi}(i) e^{\sqrt{-1} i k}
$$

and therefore recursively for all $V_{j} \subset V_{j+1}$,

$$
\hat{\phi}(k)=\prod_{j=1}^{+\infty} \hat{F}_{\phi}\left(2^{-j} k\right) \text {. }
$$

The filter $\hat{F}_{\phi}$ is $2 \pi$ periodic and satisfies:

$$
\left\{\begin{array}{l}
\hat{F}_{\phi}(0)=1 \text { to ensure } L^{2} \text {-norm normalization, } \\
F_{\phi}(i \rightarrow \infty)=\mathcal{O}\left(i^{-2}\right) \text { in order for } \phi \text { to decay at infinity, } \\
\left|\hat{F}_{\phi}(k)\right|^{2}+\left|\hat{F}_{\phi}(k+\pi)\right|^{2}=1
\end{array}\right.
$$

in order for $\hat{F}_{\phi}$ to be a conjugate filter. 


\section{FARGE}

The smoothness of the scaling function $\phi$ and its asymptotic decay at infinity can be estimated from the properties of $\hat{F}_{\phi}(k)$.

For instance, we can characterize the Meyer orthogonal wavelets by their associated discrete filters:

$$
\hat{\phi}(k)= \begin{cases}1 & \text { if } 0 \leq|k| \leq \frac{\pi}{3} \\ 0 \leq \hat{F}_{\phi}(k) \leq 1 & \text { if } \frac{\pi}{3}<|k|<\frac{2 \pi}{3} \\ 0 & \text { if }|k| \geq \frac{2 \pi}{3} \\ \left|\hat{F}_{\phi}(k)\right|^{2}+\left|\hat{F}_{\phi}(1-k)\right|^{2}=1 & \forall k\end{cases}
$$

with

$$
\hat{F}_{\phi}(k)=\cos \left[\frac{\pi}{2} \alpha(k)\right]
$$

and

$$
\begin{cases}\alpha(k)=0 & \text { if } 0 \leq|k| \leq \frac{\pi}{3} \\ \alpha(k)+\alpha(\pi-k)=1 & \text { if } \frac{\pi}{3}<|k|<\frac{2 \pi}{3} \\ \alpha(k)=1 & \text { if }|k| \geq \frac{2 \pi}{3} \\ \alpha(k)=\alpha(-k) & \forall k .\end{cases}
$$

Meyer wavelets are very regular $\left(C^{\infty}\right)$ but not very well localized in physical space. Their decrease at infinity depends on the smoothness of function $\alpha(k)$; if $\alpha$ is $C^{\infty}$, the associated Meyer wavelet will have a fast decay. There are other orthogonal wavelets, based on spline functions of order $m$, which are better localized, with an exponential decay, but which are consequently less regular, only $\mathrm{C}^{m-2}$; this is the case for instance with the Battle-Lemarié wavelets (Figure $5 a$ ).

The Mallat algorithm implies the existence of another discrete filter $F_{\psi}$ associated with the wavelet $\psi$ and in quadrature with $F_{\phi}$, namely such that the filter $F_{\phi}$ associated with the scaling function $\phi$ is a low-pass filter, while the filter $F_{\psi}$ associated with the wavelet $\psi$ is a band-pass filter (Figure $4 b)$.

We can then compute the scaling function $\phi$ and its associated wavelet $\psi$ from the discrete filter $F_{\phi}$ alone: 


$$
\begin{aligned}
& \phi(x)=\sum_{i} F_{\phi}(i) \phi(2 x-i) \\
& \psi(x)=\sum_{i} F_{\psi}(i) \phi(2 x-i)
\end{aligned}
$$

with

$$
F_{\psi}(i)=(-1)^{1-i} F_{\phi}(1-i) \text {. }
$$

In practice, to compute the wavelet transform it is only necessary to know the coefficients of the discrete filter $F_{\phi}(i)$ because, due to the quadrature condition, $F_{\psi}(i)$ is deduced from $F_{\phi}(i)$ as in Equation (46).

The algorithm (explained in detail in Mallat 1989a, Daubechies 1989, Méneveau 1991a) then consists of a pyramidal succession of discrete convolutions of the signal $f$ discretized into $I=2^{j}$ samples (Figure 4c), first with $F_{\phi}$ to compute the coefficients $\vec{f}_{j}$ describing the large-scale behavior of $f$ up to the scale $l=2^{-j}$, and secondly with $F_{\psi}$ to compute the wavelet coefficients $\widetilde{f}_{f}$ describing the behavior of $f$ around the scale $l$, such as

$$
\begin{aligned}
& \bar{f}_{j i}=\sum_{i} F_{\phi}\left(i^{\prime}-2 i\right) \bar{f}_{j-1, i} \\
& f_{j i}=\sum_{i^{\prime}} F_{\psi}\left(i^{\prime}-2 i\right) \bar{f}_{j-1, j^{\prime}}
\end{aligned}
$$

This algorithm is pyramidal in the sense that, scale after scale and from the small scales to the large scales, we undersample the signal by taking one sample out of every two smaller scale samples; therefore the number of wavelet coefficients is divided by two at each scale. Finally, due to the orthogonality of the wavelet transform, we obtain the same number $I$ of wavelet coefficients as the number of samples of the signal. From those wavelet coefficients $\bar{f}_{0}, \tilde{f}_{0} \ldots \tilde{f}_{s}$, we can then reconstruct a discrete approximation of the signal at a given scale $2^{-j}$, by the same succession of convolutions with $F_{\phi}$ and $F_{\psi}$, scale after scale but now going from the large scales to the scale $2^{-j}$. Traversing down the pyramid, we must now oversample the wavelet coefficients by inserting a zero between successive coefficients, scale after scale. To finally obtain the discrete approximation at scale $2^{-j}$, we add, following Equation (36), both results, $\vec{f}_{j-1}$ obtained from $F_{\phi}$ and $f_{j-1}$ obtained from $F_{\psi}$. For both analysis and reconstruction the operation count of the Mallat algorithm varies as $I \log _{2} I$.

As for the continuous wavelet transform algorithm (Section 3.3), we should beware of boundary effects; these are discussed for instance in Méneveau (1991a). We may also prefer to use periodic orthogonal wavelets, for which a fast algorithm similar to Mallat's has been developed by Perrier (Perrier \& Basdevant 1989), or to use the new orthogonal wavelet bases proposed by Jaffard \& Meyer (1989) which vanish on the boundaries, but for which there does not yet exist any fast algorithm. 


\section{FARGE}

$\phi(x)$
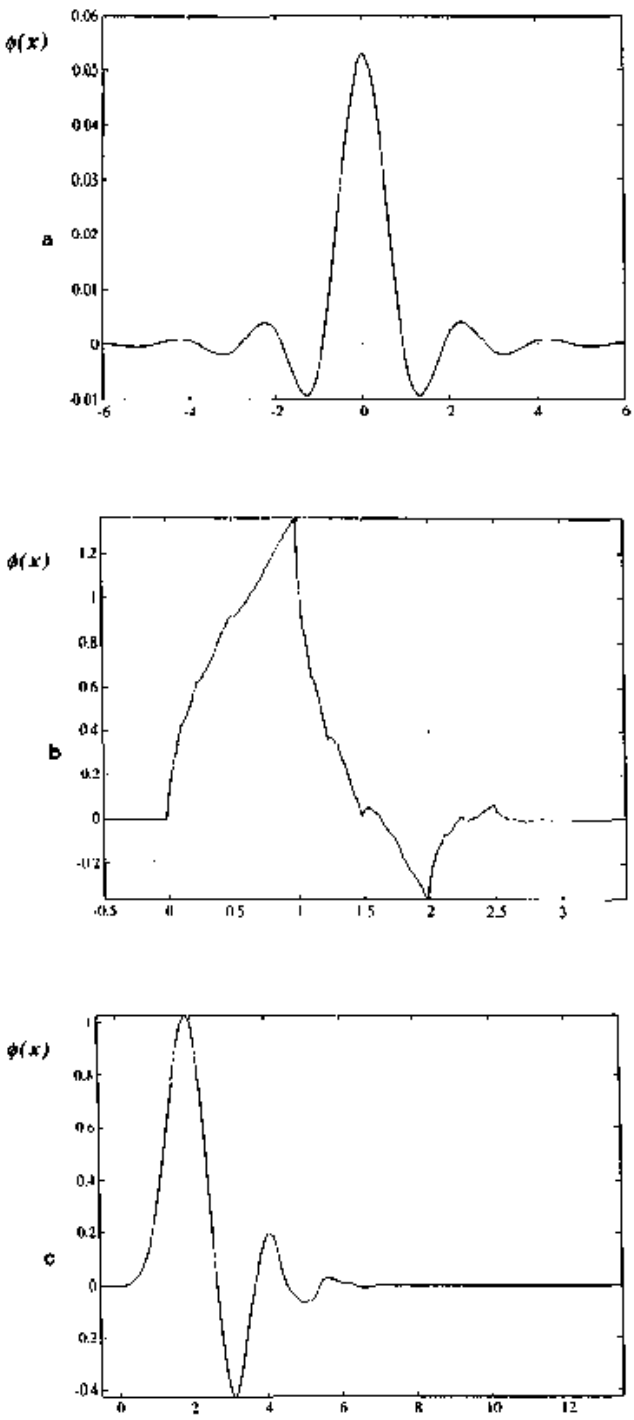

$\hat{\phi}(k)$

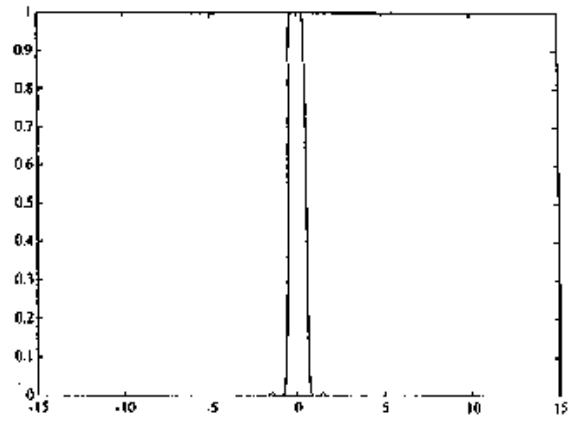

$\hat{\phi}(k)$

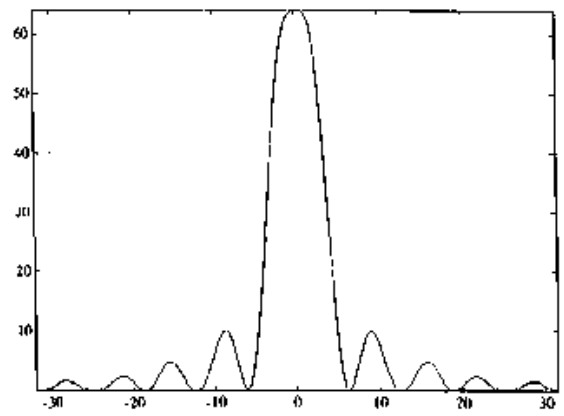

$\hat{\phi}(k)$

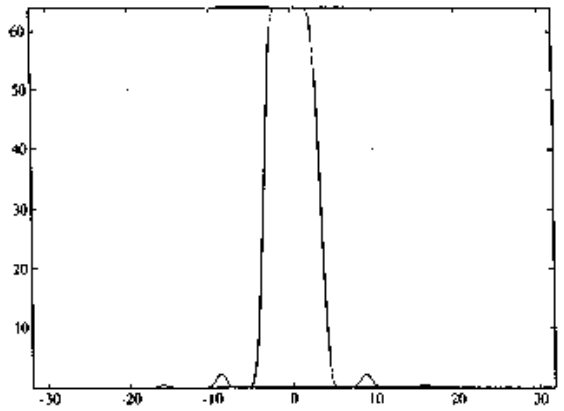

Figure 5 Examples of scaling functions $\phi$ and associated wavelets $\psi$ commonly used for the orthogonal wavelet transform. We visualize $\phi(x), \phi(k), \psi(x)$, and $\psi(k)$. (a) BattleLemarié wavelet constructed with 4 th order sptine functions, (b) Daubechies compactly supported wavelet for $\mathrm{N}=2$, (c) Daubchies compactly supported wavelet for $\mathrm{N}=7$. 


\section{Annual Reviews}

www.annualreviews.org/aronline

WAVELET TRANSFORMS

425
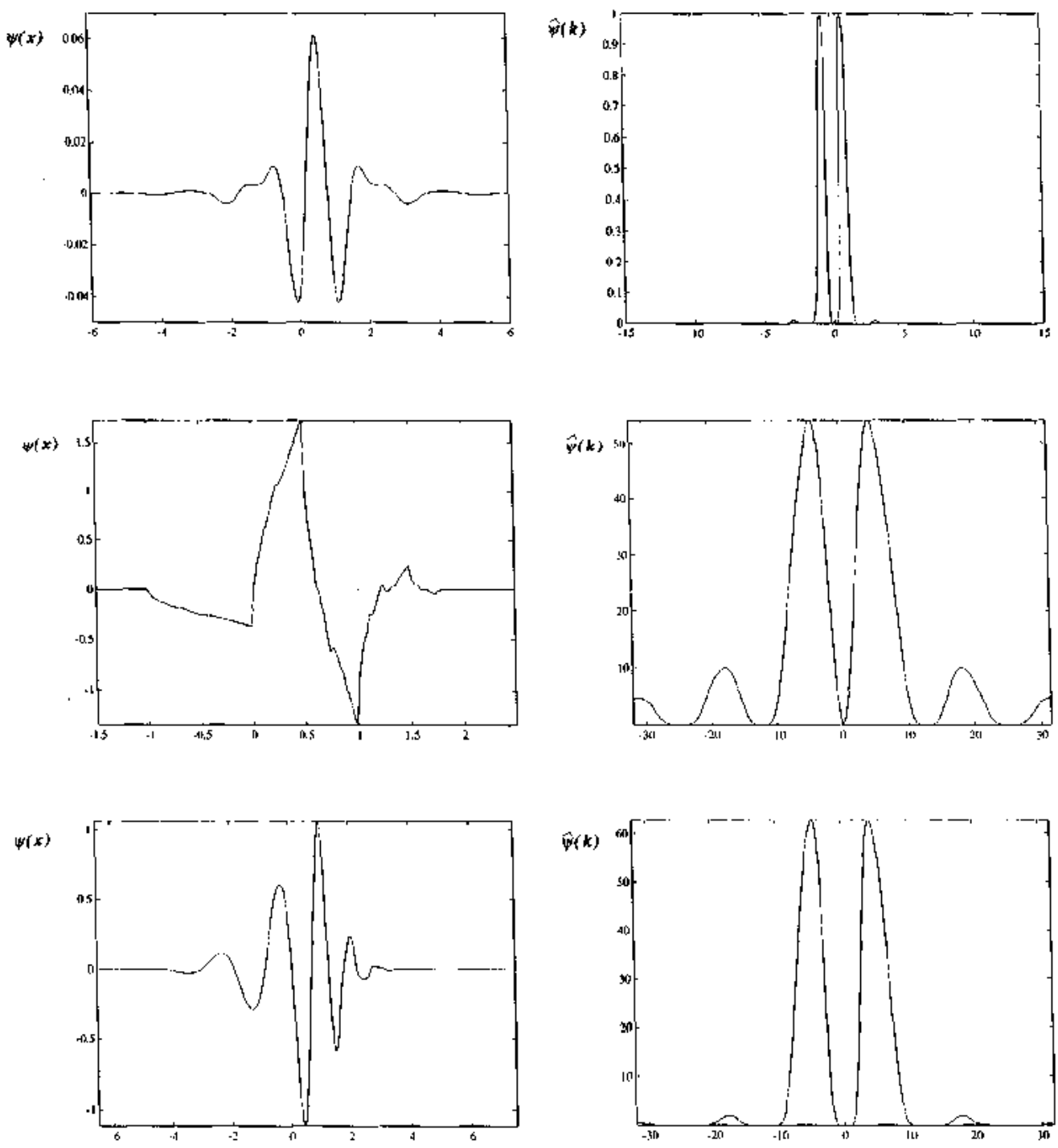

Figure 5-continted 


\section{FARGE}

COMPACTLY SUPPORTED WAVELETS Using Mallat's procedure (43), Daubechies has constructed several compactly supported and regular wavelet bases (Daubechies 1988, 1989). She showed that $\psi$ and $\phi$ are compactly supported if

$$
\left|\hat{F}_{\phi}(k)\right|^{2}=2-\frac{(2 N-1) !}{(N-1) ! 2^{2-2 N}} \int_{0}^{k} \sin ^{2 N-1} x d x .
$$

The size of the discrete wavelct support is given by $2 N-1$ and depends on the desired regularity $m$ of the wavelet because $N \geq m+1$ (Figures $5 b, c$ ).

The case $N=1$ is the Haar basis which is not regular. But for $N \neq 1$ we obtain many orthogonal interpolation bascs $\phi_{N}$ and their associated orthogonal wavelet bases $\psi_{N}$ which are continuous and differentiable until order $m \geq N / 5$. For example in the case $N=2$ (Figure $5 b$ ), the discrete filter which generates the corresponding Daubechies basis is

$$
\begin{aligned}
& F_{\phi}(0)=\frac{1+\sqrt{3}}{4 \sqrt{2}} \quad F_{\phi}(1)=\frac{3+\sqrt{3}}{4 \sqrt{2}} \\
& \left.F_{\phi}(2)=\frac{3-\sqrt{3}}{4 \sqrt{2}} \quad F_{\phi}(3)=\frac{1-\sqrt{3}}{4 \sqrt{2}} \quad F_{\phi}(i \in]-\infty,-1\right] \cup[4,+\infty[)=0 .
\end{aligned}
$$

The numerical implementation of the orthogonal wavelet transform with Daubechies' wavelets is carried out using the Mallat algorithm. Using compactly supported wavelets the operation count, for both analysis and synthesis, then varies as $I$, which is therefore faster than the Fast Fourier Transform.

PERIODIC ORTHOGONAL WAVELETS The extension of the multiresolution analysis to the case of periodic wavelets was proposed by Meyer (1986) and performed by Perrier and Basdevant who applied it to build orthogonal wavelet bases from periodic spline functions (Perrier \& Basdevant 1989). The extension of the multiresolution analysis to manifolds such as the sphere is difficult. For instance the sphere does not have the dilation and rotation invariance of the plane. Recently Jaffard has constructed orthogonal wavelet bases adapted to spherical geometries (Jaffard 1990a, Jaffard \& Meyer 1989).

BIORTHOGONAL WAVELETS At first glance Daubechies wavelets look strange: They are not symmetric (Figures $5 b, c$ ) and for $N \leq 2$ they are left differentiable but not right differentiable (Figure $5 b$ ). Recently Vetterli and Herley have designed some biorthogonal systems of compactly 
supported symmetric wavelets built from linear phase filters (Vetterli \& Herley $1990 \mathrm{a}-\mathrm{c}$ ). The use of biorthogonal wavelets constitutes a new approach initially proposed in the context of the continuous wavelet transform by Tchamitchian $(1986,1987)$, and then in the context of the orthogonal wavelet transform by Cohen, Daubechies, and Feauveau (Feauveau 1989, 1990; Cohen et al 1990; Cohen 1990). It replaces the wavelet $\psi$ by a pair of wavelets, one used for the analysis and the other used for the reconstruction. Biorthogonal wavelets are very promising because they offer much more flexibility in the choice of wavelet than orthogonal wavelets. We can, for instance, choose the properties of both wavelets to be complementary, with high-order cancellations for the analyzing wavelet and good regularity for the synthesizing wavelet.

$N$-DIMENSIONAL ORTHOGONAL WAVELETS In contrast to the continuous wavelet transform we do not yet know how to compute an orthogonal wavelet transform using an intrinsically $n$-dimensional wavelet, but it is certainly feasible. We are presently left with a partially satisfactory solution, namely that of separating the orthogonal wavelet transform of a $n$-dimensional field into $n$ orthogonal wavelet transforms performed in each spatial direction. This variable separation approach, which can also be used for the continuous wavelet transform, is therefore intrinsically anisotropic and requires $2^{n}-1$ wavelets.

For instance, to obtain a two-dimensional multiresolution analysis we start from a one-dimensional multiresolution analysis, defined by the scaling function $\phi$, from which we deduce its associated wavelet $\psi$. Then by tensor products we obtain the two-dimensional scaling function

$$
\phi\left(x_{1}, x_{2}\right)=\phi\left(x_{1}\right) \phi\left(x_{2}\right)
$$

and the associated wavelets

$$
\begin{aligned}
& \psi_{1}\left(x_{1}, x_{2}\right)=\phi\left(x_{1}\right) \psi\left(x_{2}\right) \\
& \psi_{2}\left(x_{1}, x_{2}\right)=\psi\left(x_{1}\right) \phi\left(x_{2}\right) \\
& \psi_{3}\left(x_{1}, x_{2}\right)=\psi\left(x_{1}\right) \psi\left(x_{2}\right) .
\end{aligned}
$$

This is the approach used to extend the Mallat orthogonal wavelet algorithm to two dimensions (Mallat 1988) and to three dimensions (Meneveau 1991a).

\subsection{Wavelet Packets}

Very recently, motivated by data compression problems, Coifman, Meyer, and Wickerhauser have defined and catalogued an extensive "library" of 


\section{FARGF}

functions they called "wavelet packets," from which can be built a countable infinity of orthogonal bases of $L^{2}$ (R) (Coifman et al 1990a,b; Wickerhauser 1991). The infinitely many bases of wavelet packets unify Gabor wave packets [used in the Windowed Fourier Transform (Gabor 1946)] and wavelets into a set of localized oscillating functions of zero-mean parametrized by scale $l$, space $x$, and frequency $k: l$ corresponds to the width of their spatial support, $x$ to the position of their center, and $k$ to the number of oscillations in their spatial support. A wavelet packet family is thus generated by dilation, translation, and modulation of a "mother wavelet." The different transforms (Figure 1) can be seen as the convolution of a given signal to be analyzed with a bank of filters given by the analyzing functions: filters of constant bandwidth $\Delta k$ for the Windowed Fourier Transform or filters of constant ratio of width to center frequency $k$ for the Wavelet Transform (5). The Wavelet Packets Transform in fact combines these two approaches and offers the possibility to adjust the ratio $\Delta k / k$ of the analyzing functions to the signal to be analyzed.

In the discrete case Coifman and Meyer have derived analytic formulas to generate the $2^{i}$ wavelet packets associated with a signal sampled on $I$ points. Their wavelet packets are given as a set of $I \log _{2} I$ vectors organized into a binary tree, which drastically facilitates further computations. Then for a given signal, or for each portion of it after performing an appropriate segmentation, one can choose the most appropriate orthogonal basis to decompose it. Wickerhauser proposes to select the basis that minimizes the information entropy or the number of bits necessary to code the information content of the signal (Wickerhauser 1991, Coifman et al 1990b). In practice the best basis will be that which minimizes the number of significant (that is above a certain threshold) coefficients. Therefore the wavelet packet transform of a signal of length $I$ gives at most $I$ coefficients (in general many fewer) from which the signal can be resynthesized. The analysis requires $I \log _{2} I$ operations and the synthesis $I$ operations. The wavelet packet decomposition gives orthogonal bases quite similar to those obtained with the Karhunen-Loève decomposition, or Proper Orthonormal decomposition (Lumley 1981, Aubry et al 1988), but its computing cost is much less; indeed the Karhunen-Loève decomposition requires the computation of the eigenfunctions of the correlation matrix, which contains $I^{3}$ coefficients.

Torrésani has proposed a generalization of the wavelet packet transform to the continuous case in which the wavelet packets are indexed by a continuous parameter (Torrésani 1991). The wavelet transform in fact adapts the tiling of the phase-space (Figure 1) to each portion of the signal. For instance, in regions dominated by harmonic behavior it will choose the most appropriate Gabor wave packet basis (Figure 1c), while in 
regions with strong transients or shocks it will choose the most appropriate wavelet basis (Figure 1d). Another construction which involves wavelet packets with discrete scale parameters and continuous translation parameters has recently been proposed by Duval-Destin et al (1991).

\section{WAVELET APPLICATIONS TO TURBULENCE}

\subsection{Energy Decomposition}

A very common pitfall when using any kind of transform is to forget the presence of the analyzing function in the transformed field, which may lead to severe misinterpretations, the structure of the analyzing function being interpreted as characteristic of the phenomena under study. To reduce this risk we should choose the analyzing function in accordance to the intrinsic structure of the field to be analyzed. For instance the trigonometric functions used in the Fourier transform would be the appropriate tool if and only if a turbulent flow field were a superposition of waves; only in this case are wavenumbers well defined and the Fourier energy spectrum meaningful for describing and modeling turbulence. If, on the contrary, turbulence were a superposition of point vortices then the Fourier spectrum in this case would be meaningless. The problem we still face in turbulence theory is that we have not yet identified the typical "objects" that compose a turbulent field. Before developing a turbulence model we must identify these elementary "objects" and catalog their elementary interactions. For instance the cascade models (Desnjanski \& Novikov 1974, Kraichnan 1974, Frisch \& Sulem 1975, Bell \& Nelkin 1978, Gledzer et al 1981) assume that wavenumber octaves are the elementary objects needed to describe homogeneous turbulence and that their interactions consist of exchanging energy with the neighboring octaves.

The first step toward modeling turbulence is to find an appropriate segmentation of the energy density in $x-l$ phase space (Figure 1) and to define some kind of phase-space "atoms" among which energy, or any other dynamically relevant quantity, is distributed and exchanged by the turbulent flow dynamics. If a turbulent field is a superposition of waves, the energy density should be distributed in phase space among horizontal bands, each band corresponding to an excited wavenumber (Figures $2 b, c$ ). If a turbulent field is a set of localized structures-often called coherent structures-the energy density should be distributed among cone-like patterns, each cone pointing to an excited structure (Figure $2 a$ ). If a turbulent field is a superposition of wavepackets, such as Tennekes and Lumley's eddies (Tennekes \& Lumley 1972), the energy density should be distributed among patches whose horizontal length will correspond to the 
spatial support and vertical length to the bandwidth characterizing each excited wavepacket. If a turbulent field is mainly some kind of noise, its energy density should be randomly distributed in both space and scale without presenting any characteristic pattern in phase space (Figure $2 c$ ). Actually a turbulent field may well be the superposition of different phase-space structures which can be separated into characteristic classes. In this case it will be more appropriate to docompose the flow field into those classes and then perform separate ensemble or time averages, class by class, in order to retain as much dynamically meaningful information as possible on the flow. Another solution is to perform the averaging directly in phase space, which presents the advantage of being able to add together experimental data of different signal-to-noise ratios or numerical fields computed at different resolutions. It also may well be that different types of turbulence (e.g. boundary layer, mixing layer, grid turbulence ... ) or different regimes (e.g. transition, fully-developed turbulence), will lead to different segmentations of phase space; this should be checked and, if it is the case, we should probably abandon the quest for a universal theory of turbulence.

Finally we should study how the turbulent dynamics transports these space-scale "atoms," distorts them, and exchanges their energy during the flow evolution. Using a space-scale representation it would then be easy to verify whether energy transfers are either mainly local in scale-as assumed by the cascade models - corresponding to vertical translations in phase-space, or whether energy transfers are instead local in space as assumed by point vortex models --corresponding to horizontal translations in phase-space. Energy transfers may in fact be local in both space and scale, which is probably the right answer. Wavelets or wavelet packets arc certainly good candidates for performing this energy decomposition in phase space and for finding possible phase-space atoms to characterize the turbulent flow dynamics and hence to formulate new turbulence models.

\subsection{New Diagnostics for Turbulence}

Before discussing the actual applications of wavelets to turbulence, let us emphasize two points. First of all, wavelets are useful as a new diagnostic tool for the study of turbulence if we want to retain some information about the spatial structure of the flow. If we are only interested in its frequency content, or if we want to filter it everywhere in space, wavelets are not helpful, and the Fourier transform is a sufficient tool. Secondly, we should always bear in mind the fact that wavelets see signal variations but are blind to constant and other global polynomial behavior, according to the number of cancellations (Section 3.2) of the analyzing wavelet. A 
common pitfall in interpreting wavelet coefficients is to link their strength to the signal's strength, whereas they actually correspond to variations in the signal at a given scale and a given point. If the signal does not oscillate at a certain scale and position, then the corresponding wavelet coefficients are zero.

For the purpose of analysis we prefer to use the continuous wavelet transform whose redundancy allows an unfolding of the flow information on the space-scale complete grid, as opposed to the dyadic grid used for orthogonal wavelets. As we have already said (Section 3.3), we strongly advise using a progressive complex-valued wavelet, because, due to the quadrature between the real and imaginary parts of the wavelet, we can then eliminate spurious oscillations of the wavelet coefficients by visualizing their modulus, instead of their real part (Figure 2). If we analyze a two-dimensional field using a two-dimensional wavelet we have at least a three-dimensional coefficient space to visualize. If the coefficients are real and therefore oscillate at all scales and locations, their graphical representation is very complicated and difficult to interpret, whereas the modulus only follows the signal energy density variations without presenting spurious oscillations.

The squared wavelet coefficient $|f(l, \mathbf{x}, \theta)|^{2}$ measures the energy level, or excitation, of a given field $f(\mathbf{x})$ in terms of space, scale, and direction. Let us consider the case of three-dimensional turbulence and analyze threedimensional fields in terms of three space dimensions $\mathrm{x}=x_{n}$ and three angles $\theta=\theta_{n}$, for $n=1$ to 3 . By choosing an isotropic wavelet, or averaging over directions, we can discard the angular selectivity of this analysis. This is what we shall do from now on in order to simplify the notation. We will now list several new diagnostics, for two $(n=2)$ or three $(n=3)$ dimensional turbulence, all based on wavelet coefficients.

LOCAL WAVELET ENERGY SPECTRUM First of all the notion of "local spectrum" is antinomic and paradoxical when we consider the spectrum as a decomposition in terms of wavenumbers for, as we have previously said, they cannot be defined locally. Therefore a "local Fourier spectrum" is nonsensical because, either it is non-Fourier, or it is nonlocal. There is no paradox if instead we think in terms of scales rather than wavenumbers.

Using the wavelet transform, let us define the space-scale energy density:

$$
E(l, \mathbf{x})=\frac{|\tilde{f}(l ; \mathbf{x})|^{2}}{l^{n}}
$$

As proposed by Moret-Bailly et al (1991) in the context of turbulence, the scale decomposition in the vicinity of location $x_{0}$ is given by 
432 FARGE

$$
E_{\chi}\left(l, \mathbf{x}_{0}\right)=l^{-1} \int_{\mathbf{R}^{n}} E(l, \mathbf{x}) \chi\left(\frac{\mathbf{x}-\mathbf{x}_{0}}{l}\right) d^{n} \mathbf{x},
$$

$\chi$ being a function of finite support $\left[\mathrm{x}_{1}, \mathbf{x}_{2}\right]$, which takes into account all coefficients inside the influence cone (Section 3.2) of $x_{0}$, such that:

$$
\int x(\mathbf{x}) d^{n} \mathbf{x}=1
$$

If we choose the window $\chi$ to be a Dirac function, then the local wavelet energy spectrum becomes

$$
E_{\hat{\delta}}\left(l, \mathbf{x}_{0}\right)=\frac{\left|f\left(l, \mathbf{x}_{0}\right)\right|^{2}}{I^{n}}
$$

By integrating (51) we obtain the local energy density:

$$
E(\mathbf{x})=C_{\psi}^{-1} \int_{0^{+}}^{+\infty} E(l, \mathbf{x}) \frac{d l}{l}
$$

GLOBAL WAVELET ENERGY SPECTRUM The global wavelet spectrum is

$$
E(l)=\int_{\mathbf{R}^{n}} E(l, \mathbf{x}) d^{n} \mathbf{x}
$$

It can also be expressed in terms of the Fourier energy spectrum $E(\mathbf{k})=|\hat{f}(\mathbf{k})|^{2}$ using relation (7):

$$
E(l)=\int_{\mathbf{k}^{n}} E(\mathbf{k})|\hat{\psi}(l \mathbf{k})|^{2} d^{n} \mathbf{k} .
$$

This shows that the global wavelet energy spectrum corresponds to the Fourier energy spectrum smoothed by the wavelet spectrum at each scale.

We can then recover the total energy of the field $f(\mathbf{x})$ :

$$
E=C_{\psi}^{-1} \int_{0^{+}}^{+\infty} E(l) \frac{d l}{l}
$$

LOCAL INTERMITTENCY MEASURE To measure intermittency, namely the fact that energy at a given scale may not be evenly distributed in space, Farge et al (1990) have defined the intermittency measure:

$$
I(l, \mathbf{x})=\frac{|\tilde{f}(l, \mathbf{x})|^{2}}{\left\langle|\widetilde{f}(l, \mathbf{x})|^{2}\right\rangle_{\mathbf{x}}}
$$

$I(l, \mathbf{x})=1, \forall \mathbf{x}$ and $\forall l$, means that there is no flow intermittency, i.e. that 
each location has the same energy spectrum, which then corresponds to the Fourier energy spectrum. $I\left(l, \mathbf{x}_{0}\right)=10$ means that the point $\mathbf{x}_{0}$ contributes 10 times more than the average over $\mathbf{x}$ to the Fourier energy spectrum at scale $l$.

Frick \& Mikishev (1990) had previously defined a similar intermittency measure using the Zimin hierarchical model (Zimin 1981).

SPACE-SCALE REYNOLDS NUMBER AND GLOBAL INTERMITTENCY MEASURE Farge et al (1990b) have introduced a space-scale Reynolds number

$$
\operatorname{Re}(l, \mathbf{x})=\frac{\tilde{v}(l, \mathbf{x}) l}{v}
$$

where $v$ is the kinematic viscosity of the fluid and $\tilde{v}$ the characteristic rms velocity at scale $l$ and location $\mathbf{x}$ such as:

$$
\tilde{v}(l, \mathbf{x})=\left[\left(3 C_{t p}\right)^{-1} \sum_{n=1}^{3}\left|\tilde{v}_{n}(l, \mathbf{x})\right|^{2}\right]^{1 / 2} .
$$

At large scales $l \sim L$,

$$
\operatorname{Re}(L)=\int_{-\infty}^{+\infty} \operatorname{Re}(L, \mathbf{x}) d^{n} \mathbf{x}
$$

coincides with the usual large-scale Reynolds number $\operatorname{Re}=v^{\prime} L / v$, where $v^{\prime}$ is the rms turbulent velocity and $L$ the integral scale of the flow. At the Kolmogorov scale $l \sim \eta$, where dissipation effects equilibrate nonlinear effects, $\operatorname{Re}(\eta, x)=1$. In plotting the iso-surface $\operatorname{Re}(l, x)=1$ we can then check whether it is flat or not. If it is flat, then $l=\eta$ everywhere, as assumed by Kolmogorov's theory. If it is not flat, then the turbulent flow is intermittent and we can no longer define a unique Kolmogorov scale $\eta$, but only a range of scales from $\eta_{\min }$ to $\eta_{\max }$. The ratio $I(\mathrm{Re})=\eta_{\max } / \eta_{\min }$, for $\operatorname{Re}=1$, is a global measure of the flow intermittency in the dissipative range. The ratio $I(\mathrm{Re})=l_{\max }(\mathrm{Re}) / l_{\min }(\mathrm{Re})$, for $\mathrm{Re} \gg 1$, measures the global flow intermittency in the inertial range.

For direct numerical simulations of turbulent flow, this iso-surface $\operatorname{Re}(l, \mathbf{x})=1$ may be useful in detecting numerical errors and verifying if the space resolution $\Delta x$ is sufficient to resolve the dissipative scales everywhere in the flow. We have to verify that $\eta_{\min }>\Delta x$ everywhere in the flow, otherwise for points where this inequality is not verified some numerical instability may develop.

For numerical simulations we can define a better space-scale Reynolds number. For this we must compute the nonlinear term $N=\|v \cdot \nabla v\|$ and 


\section{FARGE}

the dissipative term $D=\left\|v \cdot \nabla^{2} v\right\|$ and expand them into their wavelet coefficients to obtain

$$
\operatorname{Re}(l, \mathbf{x})=\tilde{N}(l, \mathbf{x}) / \tilde{D}(l, \mathbf{x})
$$

SPACE-SCALE CONTRAST If we want to detect any variation in the signal, even for regions where the signal becomes very weak, Duval-Destin and Torrésani (Duval-Destin \& Menu 1989; Antoine et al 1990, 1991; DuvalDestin et al 1991; Antoine \& Duval-Destin 1991) have proposed the contrast measure

$$
C(l, \mathbf{x})=\frac{|\tilde{f}(l, \mathbf{x})|^{2}}{|\bar{f}(l, \mathbf{x})|^{2}},
$$

with

$$
f(l, \mathbf{x})=C_{\psi} \cdot l^{-(n+1)} \int_{r=0^{+}}^{\gamma=f} f\left(l^{\prime}, \mathbf{x}\right) d l^{\prime} .
$$

For orthogonal wavelets the contrast has the very elegant form

$$
C_{j i}=\frac{\left|\left\langle\psi_{j} \mid f\right\rangle\right|^{2}}{\left|\left\langle\phi_{j i} \mid f\right\rangle\right|^{2}}
$$

$\psi_{j i}$ being the orthogonal wavelets, at scale $j$ and location $i$, and $\phi_{j i}$ the associated scaling functions.

While wavelet coefficients locally measure the signal derivative, the space-scale contrast measures its logarithmic derivative. This diagnostic may be useful in fluid mechanics for detecting some very weak coherent structures, or some coherent structures embedded in larger ones-two cases that cannot be detected by the classical threshold method. In any case wavelets are better adapted to filtering or segmenting flow fields than traditional image processing techniques; most image processing techniques are based on contour detection developed for pattern recognition, whereas coherent structures encountered in fiud flows do not have sharp contours but instead are characterized by their local scaling. A similar problem is encountered in analyzing astronomical images, in particular in detecting galaxies, for which wavelets are now extensively used (Bijaoui 1991).

SPACE-SCALE ANISOTROPY MEASURE The measure of the space-scale departure from isotropy is given by 


$$
A(l, \boldsymbol{\theta})=\frac{\int_{-\infty}^{+\infty}|\tilde{f}(l, \mathbf{x}, \boldsymbol{\theta})|^{2} d^{n} \mathbf{x}}{\left\langle\int_{-\infty}^{+\infty}|\tilde{f}(l, \mathbf{x}, \boldsymbol{\theta})|^{2} d^{n} \mathbf{x}\right\rangle}
$$

For this diagnostic we obviously must use a directional wavelet such as the Morlet wavelet (Section 3.3).

If $A(l, \theta)=1, \forall l$ and $\forall \theta$, the flow is isotropic at all scales.

If $A\left(l_{\min }, \theta\right)<A\left(l_{\max }, \theta\right), \forall \theta$, with $l_{\min }$ a small scale and $l_{\max }$ a large scale, there is a return to isotropy in the small scales.

$A\left(l, \theta_{0}\right)=10$ means that direction $\theta_{0}$ contributes 10 times more than the average over $\theta$ to the Fourier energy spectrum at scale $l$.

LOCAL SCALING AND SINGULARITY SPFCTRUM As we have said in Section 3.2, the continuous wavelet transform with $L^{\mathrm{t}}$-norm (19) is used to study the local scaling of a function and to detect its possible singularities. But to see singularities, we must subtract the Taylor series of the signal, which corresponds to its polynomial, and therefore differentiable, terms. This subtraction is automatic if we choose a wavelet with sufficiently many cancellations (Section 2.2 and 3.1) to be blind to these polynomial contributions. Here again a complex-valued wavelet is preferable. To justify this choice, let us take as a counterexample the analysis of a singularity $\left|\mathbf{x}-\mathbf{x}_{0}\right|^{\alpha}, 0<\alpha<1$, located at $\mathbf{x}_{0}$, using a real-valued wavelet. If the singularity is odd and the wavelet even, or vice-versa, then the small-scale wavelet coefficients will be zero at $\mathbf{x}_{0}$ and we will not see the singularity, for it has been cancelled by the signal's, or wavelet's, zero-crossings. If we choose a progressive complex-valued wavelet, whether odd or even, the $L^{\prime}$-modulus of the wavelet coefficients will drastically increase in the small scales around the location $\mathrm{x}_{0}$ and the rate of increase will give the exponent of the singularity. The local scaling of $f(x)$ at $x_{0}$ is given by the scaling of the wavelet coefficients' $L^{1}$-modulus in $x_{0}$ in the limit of asymptotically small scales, $l \rightarrow 0$. This property has been used by Tchamitchian \& Holschneider $(1989,1990)$ to study the local regularity of the Riemann function: they have demonstrated that the Riemann function is differentiable on a dense set of points and is singular everywhere else.

To compute the singularity spectrum, we should proceed as follows. First, we plot the phase of the wavelet coefficients to locate singularitiesfor the iso-phase lines will point towards singular points. Second, we should verify that the singular point detected at $\mathbf{x}_{0}$ is isolated, namely that

$$
f\left(l \rightarrow 0, \mathbf{x}_{0}\right)<f\left(l \rightarrow 0, \mathbf{x}_{0} \pm \varepsilon\right) \text {, with }|\varepsilon| \text { very small. }
$$




\section{FARGE}

Then the exponent $\alpha\left(\mathbf{x}_{0}\right)$ of the singularity is given by the slope of the local scaling of $\log \left|\vec{f}\left(l, x_{0}\right)\right|$ for $l \rightarrow 0, \tilde{f}$ being written in $L^{1}$-norm (19). The singularity spectrum has been used to characterize fractal and multi-fractal measures (Arnéodo et al 1988, 1989; Arnéodo et al 1990; Arnéodo et al 1991), and its generalization to fractal and multi-fractal functions has been recently made by Muzy et al (1991). Using a real-valued wavelet, Bacry et al (1991) first extract the wavelet coefficients "skeleton," i.e. the location of the local maxima of the wavelet coefficients, before computing the local scaling. Benzi \& Vergassola (1991) proposed a proccdure, called "the optimal wavelet transform," to reduce possible oscillations of $\left|\tilde{f}\left(l, \mathbf{x}_{0}\right)\right|$ versus $\log /$ while using a real-valued wavelet. This procedure is computationally intensive and has been recently improved by using a progressive complex-valued wavelet (Morlet) instead of a real-valued one (Benzi \& Vergassola 1991).

\subsection{Some Preliminary Results Using Wavelets}

FOR TURBULENCE ANALYSIS Wavelet analysis of turbulent flows is at present developing rapidly in many different directions. As a consequence, I cannot detail and discuss all published papers on this subject, thus will only list those I know of, so that the reader may have access to them. As noted in the introduction, most of these papers have an exploratory character and their conclusions may not yet be definitive. They do have the merit of clarifying the concepts inherited from the Fourier spectral approach, of asking new questions, and of introducing new points of view concerning our understanding of turbulence.

To my knowledge, the wavelet transform was first used in fluid mechanics by Saracco and Tchamitchian in a study of the propagation of transient acoustic signals in inhomogeneous media (Saracco \& Tchamitchian 1988, Saracco et al 1989). In the context of turbulence, wavelets were first used by Farge and Rabreau to analyze two-dimensional homogeneous turbulent flows obtained from numerical simulations, using first a one-dimensional Morlet wavelet (Farge \& Rabreau 1988a,b; Farge \& Sadourny 1989) and then a two-dimensional Morlet wavelet (Farge \& Holschneider 1989, Farge et al 1990a, Farge 1990). They showed that during the flow evolution, starting from a random distribution of vorticity with a $k^{-3}$ energy spectrum, the small scales of the vorticity field become increasingly localized in physical space (Figure 6). This suggested an intermittency of the two-dimensional turbulence dynamics, which may be related to a condensation of the vorticity field into vortex-like coherent structures. In particular they found that the smallest scales of the vorticity field are concentrated inside some vortex cores (Figures 7,8 ) and are generated when and where two same-sign coherent structures are merging 
A Annual Reviews

R www.annualreviews.org/aronline

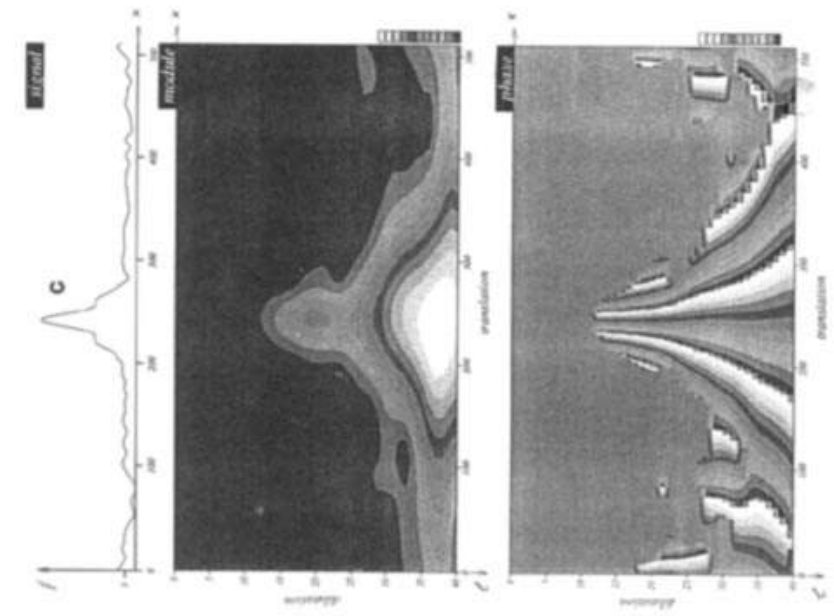

部急总

空

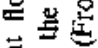

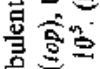

突

西照总

象里要

要

客密

要

a

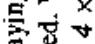

密焉 I
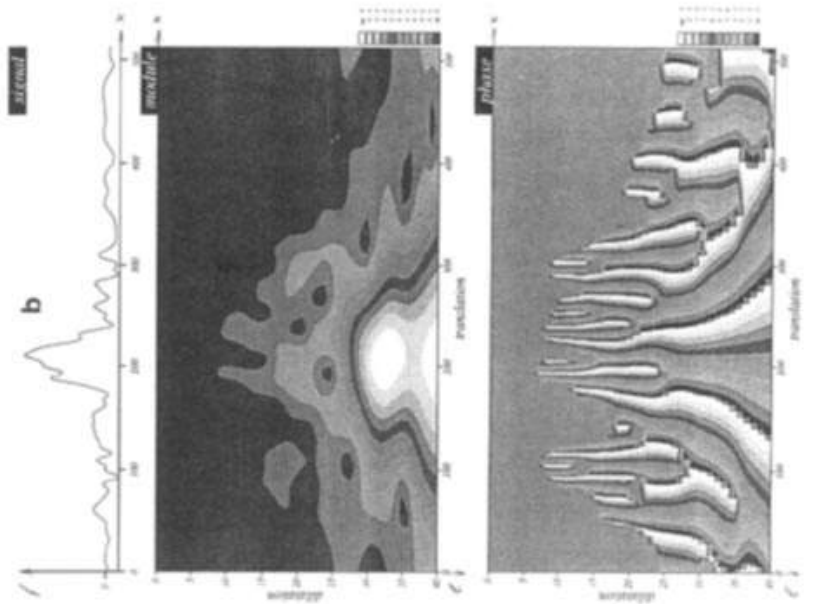

$\approx$ 的

क $1 \frac{5}{6}$

胥道

娄

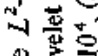

岛䒾

돈 혼

家要

宽总

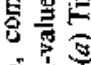

它密

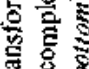
运 可高 家喓
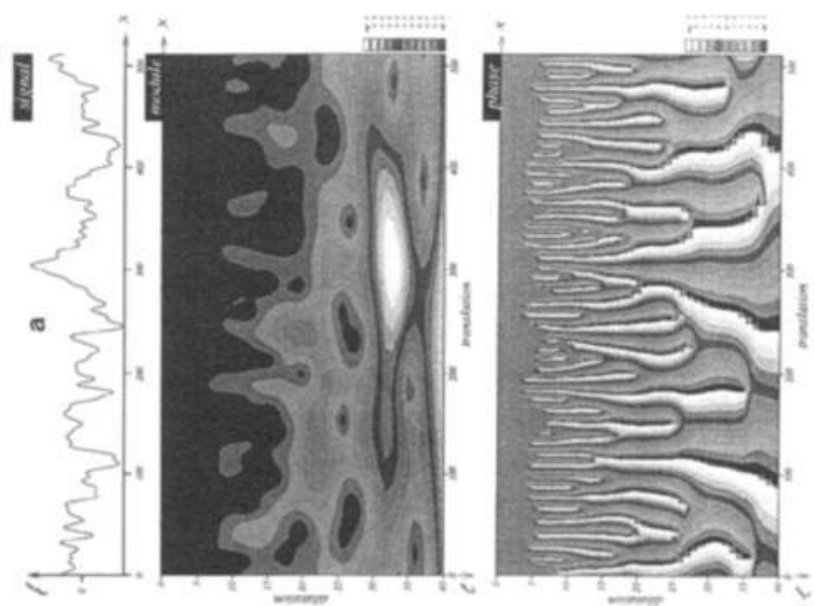

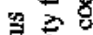

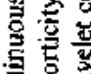

类

은

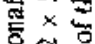

弯

的

穹宁到

总寻总离

○ 突武

6.

竞密

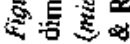


(Farge et/4:990a). From this observation, Farge and Holschneider proposed a new, purely geometrical, interpretation of the $k^{-4}$ cnergy spectrum observed for numerically computed two-dimensional turbulent flows, based on the existence of cusp-like axisymmetric coherent structures with a scaling exponent of $-1 / 2$ (Farge \& Holschneider 1991). Such quasisingular coherent structures are scaling distributions of vorticity which exhibit, instead of a characteristic radius, a range of radii corresponding to all scales of the inertial range, until the dissipative scales where the vortex cores are smoothed. These cusp-like coherent structures are also characterized by a nonlinear pointwise relation between vorticity and streamfunction, similar to coherent structures experimentally observed in a two-dimensional turbulent mercury flow (Nguyen Duc \& Sommeria 1988). Farge and Holschneider have conjectured that those quasi-singular vortices are created by the condensation of the vorticity field around quasisingularities already present in the initial conditions (Farge \& Holschneider 1991). They have also shown that coherent structures with a scaling exponent of $-1 / 2$ are stable under the two-dimensional Navier-Stokes dynamics, even when perturbed by strong noise, and these structures organize the random field in their vicinity by accreting the same-sign noise onto them (Farge et al 1991a). Recently Benzi and Vergassola, by performing a wavelet analysis of a numerically computed two-dimensional homogeneous turbulent flow, have confirmed the existence of coherent structures with negative exponents, between -0.4 and -0.6 , similar to the -0.5 scaling exponent predicted by Farge and Holschneider (Benzi \& Vergassola 1991).

The first wavelet analysis of an experimental turbulent signal was carried out by Argoul et al from the wind tunnel streamwise velocity measured at high Reynolds by Gagne and Hopfinger, using one-dimensional realvalucd wavelets (Argoul et al 1989, Arnéodo et al 1990, Bacry et al 1991, Arnéodo et al 1991). At about the same time, Liandrat, Moret-Bailly, and Tchamitchian, using a one-dimensional real-valued wavelet, studied the interaction between a shock wave and free turbulence, and then analyzed the streamwise velocity in a turbulent boundary layer near a heated wall

Figure 7 Two-dimensional continuous wavelet transform computed in the $L^{\prime}$-norm, using the real-valued DOG (Difference of Gaussians) wavelet. The same two-dimensional turbulent flow (vorticity field sampled at $\$ 12^{2}$ points) as in Figure 6 for $n=10^{3}$ has been used. We visualize the wavelet coefticients (left) at: scale $l=8 \Delta x$ (top), scale $l=4 \Delta x$ (middle), scale $l=2 \Delta x$ (botiom); where $\Delta x$ is the mesh size. We then visualize the partial reconstructions of the vorticity field (righr) from the wavelet coefficients, taking all coefficients up until: scale $l=8 \Delta x($ lop $)$, scale $l=4 \Delta x$ (middle), scale $l=2 \Delta x$ (bottom). 
A Annual Reviews

R www.annualreviews.org/aronline
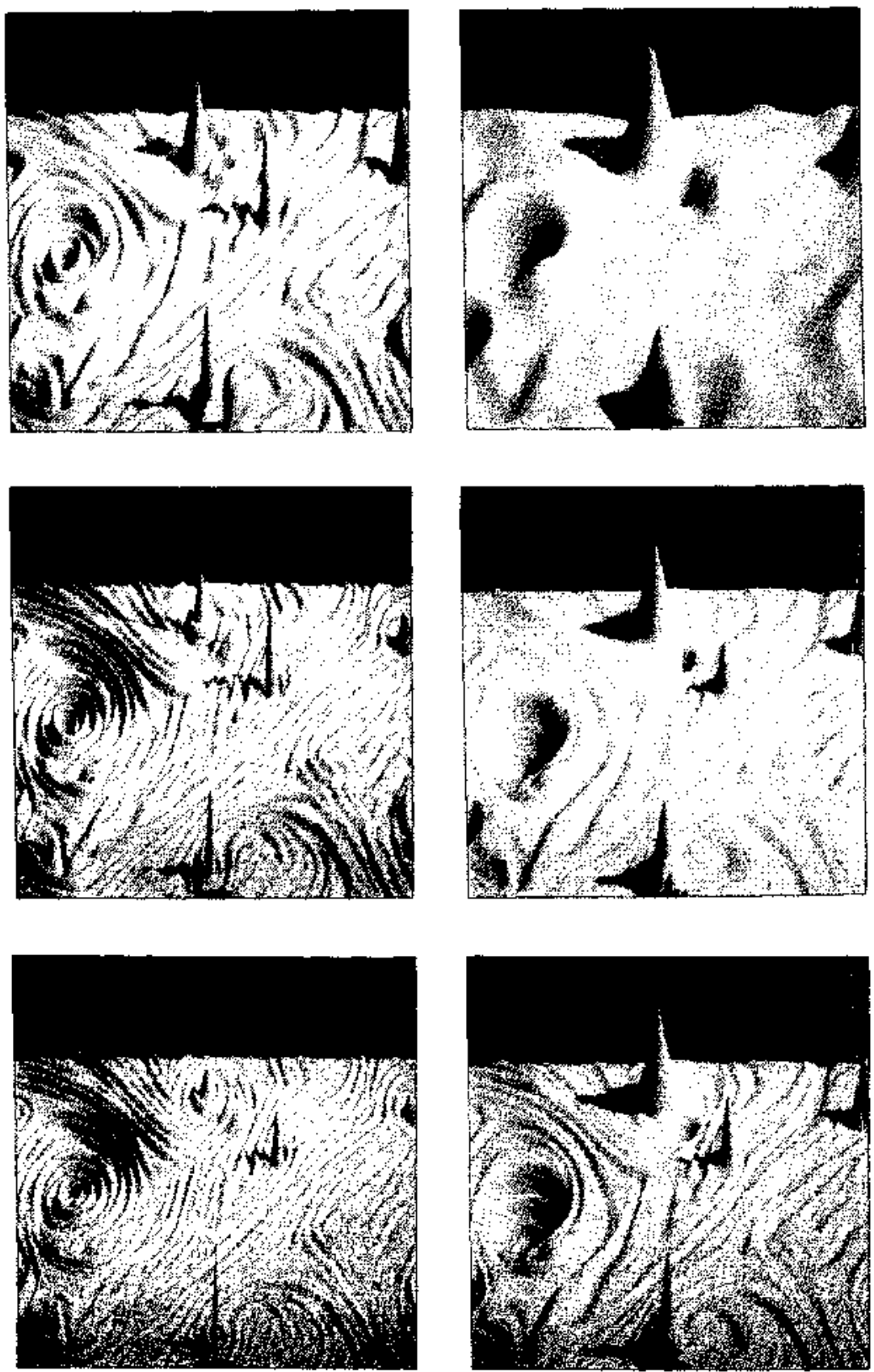


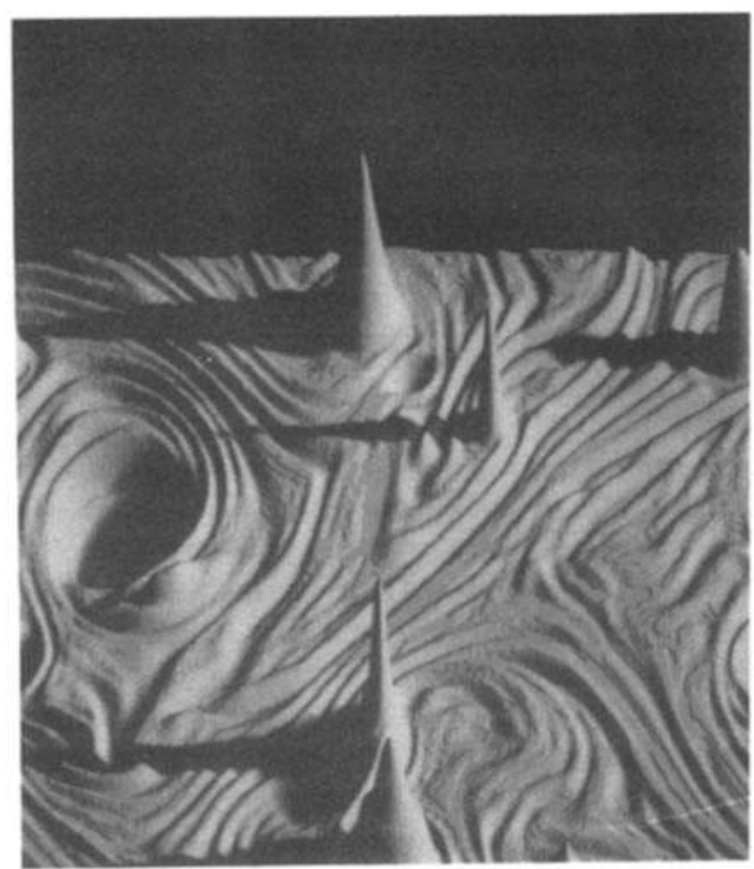

Figwre \& Two-dimensional continuous wavelet transform compled in the $L^{\prime}$-norm, using the complex-valued Motlet wavelet with $\left|k_{4}\right|=5$ and $\theta=0$, of the same two-diznensional turbolent flow (vorticity field sampled on $512^{2}$ points) as Figure 7 . The black and white figuts iliustrates the vorticity fiejo to be analyzed. The color plate shows the wavelet cocficient modulus (color coded in the increasing order: blue, red, magenta, green, cytan, yellow, and white) a rul phase (yray iso/ines) nuaped on 10 the vorticity fiejd (in perspective representation) at: scale $l=32 \Delta x$ (top) and scale $t=16 \Delta x$ (bottom). (From Farge tt al 1990a)

in order to compare both wavelet and VITA (Variable Interval Time Averaging) techniques (Liandrat \& Moret-Bailly 1990). They also used the wavelet transform to estimate the transition Reynolds number in a rotating disk boundary layer (Moret-Bailly et al 199I). More recently Frisch and Vergassola have proposed a new type of averaging for turbulence analysis, namely a scale-averaging of the logarithm of the wavelet coefficients modulus, which, like time-averaging of stationary processes, reduces statistical fuctuations of self-similar random processes, provided there is a sufficient range of scales in the signal (Vergassola \& Frisch 1990). In the study of stochastic processes and multiscale statistical signal processing, many developments are presently underway (Basseville \& Benveniste 1989, 1990; 


\section{A Annual Reviews \\ R www.annualreviews.org/aronline}
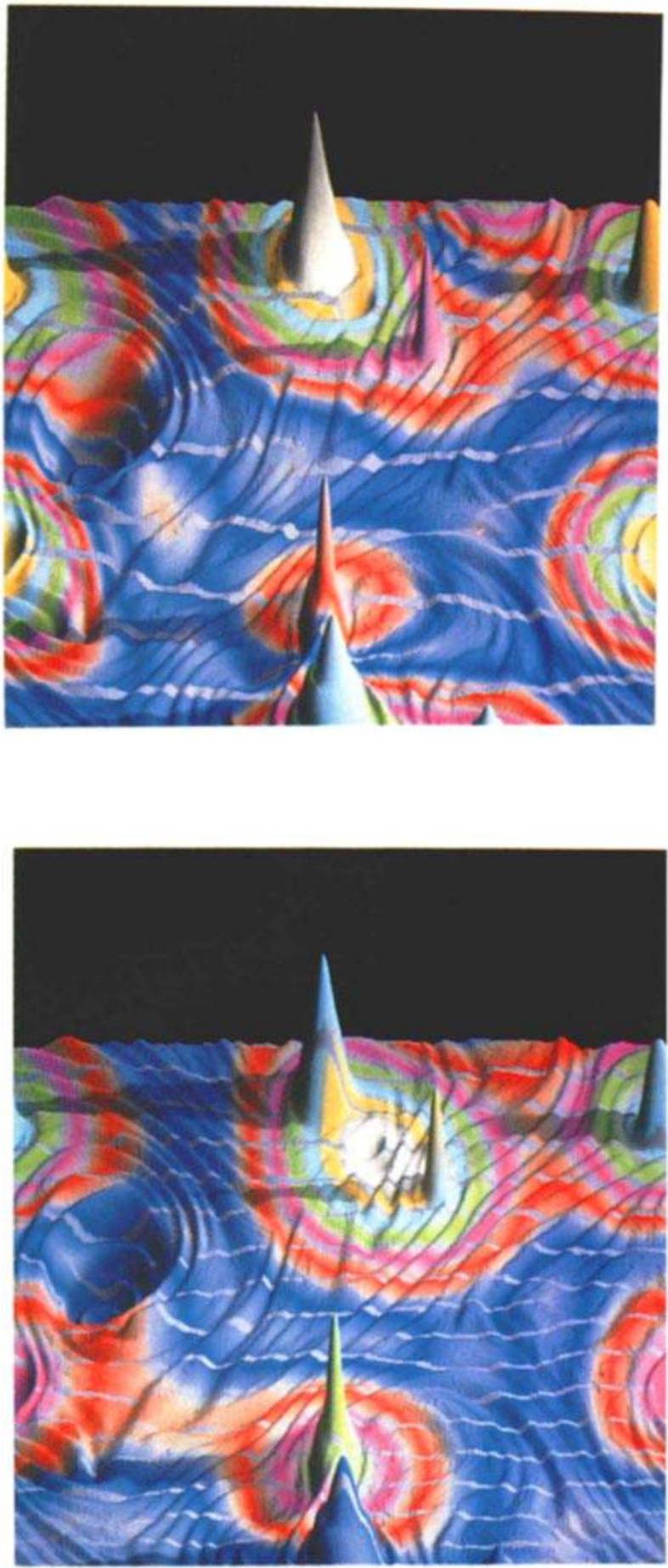
Benveniste 1990; Benveniste et al 1990; Flandrin 1988, 1990, 1991a,b; Flandrin \& Rioul 1990).

Although all the initial work [except Benzi \& Vergassola (1991)] was carried out in France, the wavelet transform has since gradually diffused abroad. In Japan, Otaguro, Takagi, and Sato have used a one-dimensional Marr wavelet (the Mexican hat) and other triangular functions (which are not actually admissible wavelets) to search for patterns in a streamwise velocity signal measured in a turbulent boundary layer (Otaguro et al 1989). Using a one-dimensional orthogonal wavelet, Yamada and Okhitani analyzed data from atmospheric turbulence (Yamada \& Ohkitani $1990 \mathrm{a}, \mathrm{b})$. In the United States, Everson and Sirovich, using different onedimensional real-valued wavelets, have computed the wavelet transform of several types of Brownian motions and of several experimental measurements: kinetic energy and Reynolds stress in the wake of a cylinder and in a numerically computed mixing layer, and kinetic energy and kinetic energy dissipation rate recorded in the atmospheric boundary layer (Everson \& Sirovich 1989). Using a two-dimensional Marr wavelet they have also compared a $k^{-2}$ Brownian motion to the dye concentration data measured by Sreenivasan in a turbulent jet at moderate Reynolds number (Everson et al 1990).

Using tensor products of Battle-Lemarié orthogonal wavelets, Meneveau has studied the energy intermittency in a wake behind a cylinder and in a boundary layer. He then computed the transfer of kinetic energy and the flux of kinetic energy through a given position and a given scale for a variety of turbulent flows: in a numerically computed homogeneous shear flow, and in an isotropic homogeneous turbulence numerical simulation (Meneveau 1991a-c). He found, first, that those quantities have nonGaussian statistics and, secondly, that the local flux of energy coming from the small scales exhibits a large spatial intermittency, and even locally presents some inverse cascades. Dallard and Spedding - using the twodimensional Halo and Arc wavelets they introduced (Section 3.3)-have analyzed Rayleigh-Bénard convection rolls and plane mixing layers in order to detect, in both space and scale, possible phase defects of those flows (Dallard \& Spedding 1990).

Using a two-dimensional Morlet wavelet, Farge, Guezennec, Ho, and Menevedu (Farge et al 1990b) analyzed different fields, such as velocity components, vorticity components, and temperature, obtained from the NASA-Ames direct numerical simulations, considering in particular the turbulent channel flow computed by Kim and Moin and the temporally evolving mixing layer computed by Rogers and Moser. They measured, using the diagnostics defined in Section 5.2, a very strong space intermittency in the small scales. They related it to the bursts ejected from the 


\section{2}

\section{FARGE}

boundary layer of the channel flow and to the ribs (streamwise vortex tubes) stretched and engulfed into the spanwise vortex cores in the case of the mixing layer. Extrapolating the local scalings they found, and considering their strong variance from the Fourier spectrum, they conjectured that the larger the Reynolds number the larger the degree of intermittency. They have also observed a return to isotropy in the small scales for the mixing layer, but not for the plane channel flow whose small scales remain elongated in the streamwise direction. Finally, they found that the isoReynolds manifold represented in space and scale (see Section 5.2) is not flat, but presents peaks in the most unstable regions, particularly in the spanwise vortex cores of the mixing layer.

Farge et al (Farge 1991c, Farge et al 1991c) have shown that the wavelet packet bases are much more efficient than the Fourier basis to compress two-dimensional turbulent flows. They define the "best basis" as the one which condenses the $L^{2}$-norm (energy or enstrophy) into a minimum number of non-negligible wavelet packet coefficients. They have found that the most significant coefficients of the best basis correspond to the coherent structures, while the weakest coefficients, which can then be discarded, correspond to the vorticity filaments (Figure 9). They have then performed several numerical integrations initialized, either with a noncompressed vorticity field (reference history), either with a vorticity field compressed using the Fourier basis, or with a vorticity field compressed using the best basis. For a compression ratio $1 / 2$, done in the best basis, the time evolution during 6000 time steps of the vorticity field remains similar to the reference history, while if the compression ratio is done in the Fourier basis, the deterministic predictability is lost and only the spectral behavior remains similar. Then for compression ratios up to $1 / 200$, done in the best basis, the deterministic predictability is lost but not the statistical predictability, whereas the compression operated in Fourier loses both. This confirms the fact, predicted among others by Farge (1990), that the dynamically active entities of a two-dimensional turbulent flow are the coherent structures, while the vorticity filaments are only passively advected by them. Using the wavelet packet best basis the flow separation into active versus passive components, or "master" versus "slave" modes, is performed without assuming any hypothetical scale separation, as is necessary when using the Fourier basis. In conclusion, the wavelet packet best basis seems to distinguish the low-dimensional dynamically active part of the flow (the coherent structures) from the high-dimensional passive components (the vorticity filaments), which can then be neglected or parametrized. This gives us some hope of drastically reducing the number of degrees of freedom necessary to compute two-dimensional turbulent flows.

To conchude this section on turbulence analysis using wavelets, I would 

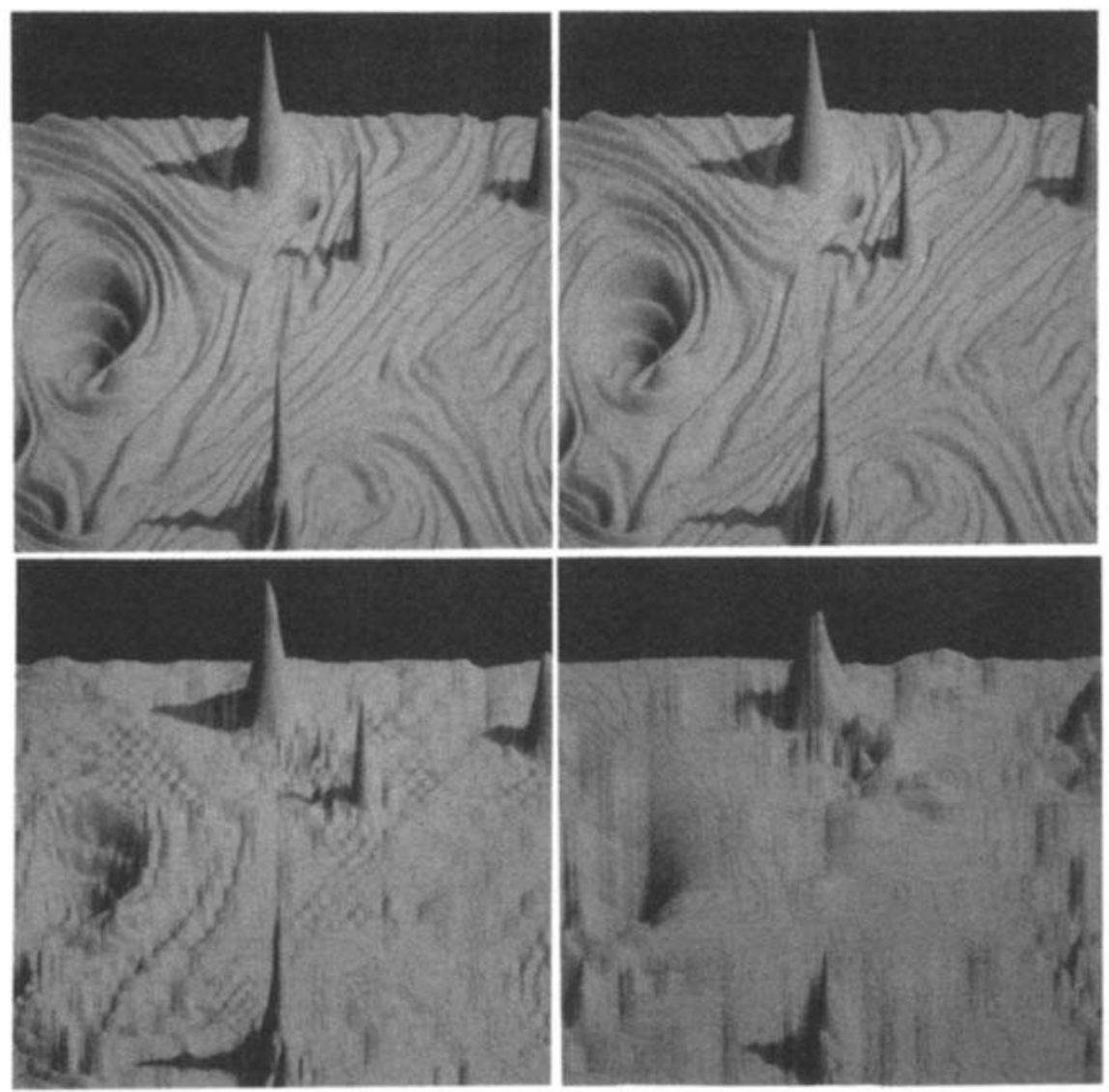

Figure 9 Two-dimensional real waveles packet transform computed in the $L^{2}$-norm of the same two-dimensional turbulent flow (vorticity field sampled at $512^{2}$ points) as in Figures 7 and 8 . Wc visualize: the vorticity field to be analyzed ( $t o p$ left). We then visualize the partial reconstructions of the vorticity field from the wavelet packet coefficients, taking only the first: $10^{4}$ most significant coefficients (top right), $10^{3}$ most significant coefficients (bottom left), 100 most significant coefficients (bottom right). (From Farge et al 1991c.)

like to quote the wise remarks of Otaguro et al (1989) as a reemphasis of what $I$ have already said in Section 5.1:

It is important to notice that if we choose a particular wavelet, the resultant correlation pattern will obediently reflect the characteristics of the wavelet. The fact may be temed sensitivity to reference. Thus we have encountered an old issue in pattern search, arbitrariness of reference. There are two points to be mentioned in this context. The first one is that the sensitivity to reference should be utilized as much as possible so that 


\section{FARGE}

we can search any object in a chaotic field. The second one, which can be contradictory to the first, however, is that we have chances to capture ghost patterns with no physical significance. This danger has been repeatedly mentioned by many authors. The problem is deeply retated to our a priori knowledge about the turbulent field under test.

FOR TURBULENCE MODELING The goal of turbulence modeling using wavelets is to directly compute the time evolution of a turbulent flow in terms of wavelet coefficients, instead of space variables. If the turbulent dynamics is governed by some nonlinear cascades, then we should study them in both space and scale, without assuming, for instance, any scale locality of the transfers as is done for the so-called cascade turbulence models (Desnjanski \& Novikov 1974, Kraichnan 1974, Frisch \& Sulem 1975, Bell \& Nelkin 1978, Gledzer et al 1981, Qian 1988). After writing Euler or Navier-Stokes equations in wavelet space, the problem is then to define the graph of possible interactions, to estimate their propagation speed in phase space, and to ascertain if transfers are mainly local in spacecorresponding to horizontal shifts in phase space, local in scale-corresponding to vertical shifts, or local in both-if shifts operate along diagonals. In studying the symmetries of the Euler or Navier-Stokes equations, and perhaps some variational principles, we might then learn which kind of interactions dominate and which are forbidden. With this approach to the modeling of turbulence, no statistical hypotheses are needed because we directly study a set of algebraic equations obtained by projecting Navier-Stokes or Euler equations onto an appropriate orthogonal basis. In this case the whole endeavor will consist of truncating as much as possible the number of algebraic equations retained in the turbulence model. As a first step in this direction, Meneveau (1991c) derived the threedimensional Navier-Stokes equation in wavelet space. His paper actually focuses on turbulence analysis (see previous paragraph), but he mentions that further developments of this formulation should concentrate on possible approximations to the interaction kernel, similar to the work of Nakano (1988) who used a wave packet formulation.

In the same spirit (but before the wavelet theory of Morlet-GrossmannMeyer) extremely impressive work was carried out by Zimin starting in 1981 in Perm, Soviet Union (Zimin 1981). In his first paper, he projected the three-dimensional Navier-Stokes equation onto a quasi-orthogonal basis using functions that were localized in both space and scale. To construct his basis he considered a hierarchy of eddies and assumed that the small eddies were advected by the larger ones. This led him to segment the phase space into cells of constant size corresponding to eddies whose spatial support decreased with the scale, each eddy having a space-scale resolution in accordance to the uncertainty principle. In fact the phasespace segmentation of Zimin's hierarchical basis is the same as for wavelets 
(Figure 1d). After constructing hierarchical bases for three-, two-, and one-dimensional flows (Zimin 1981, Frick 1983, Zimin et al 1986, Zimin \& Frick 1988, Frick \& Zimin 1991), Zimin is presently generalizing them to three dimensions plus time (Zimin 1990a,b) in order to be able to compute the Navier-Stokes equation in a purely algebraic fashion. Since 1981 Zimin's approach has been extensively used in more than 40 papersunfortunately most of them have not yet been translated from Russian. Here I only briefly list some of those, translated into English, which use the hierarchical basis for modeling or computing different types of turbulent flows: two-dimensional flows (Frick 1983, Aristov et al 1989, Mikishev \& Frick 1990), two-dimensional MHD flows (Frick 1984, Mikishev \& Frick 1989, Aristov \& Frick 1990), rotating shallow-water flows (Aristov \& Frick 1988a, Aristov et al 1989), and different convective flows (Frick 1986a,b; Frick 1987; Aristov \& Frick 1988b, 1989).

We should also mention a recent model of turbulence intermittency (Farge \& Holschneider 199l, Farge 199l b), differing from previous models (Kolmogorov 1961, Mandelbrot 1974, Frisch et al 1978, Benzi et al 1984, Parisi \& Frisch 1985), which all refer to hypothetical stochastic proccsses and often involve, for instance, eddy breaking. This new model proposes a purely geometrical interpretation of intermittency: spatial intermittency, for both two-dimensional and three-dimensional turbulent flows, may be related to the quasi-singular shape of a few highly excited axisymmetric coherent structures, which are produced by the flow dynamics. Due to their cusp-like shapc, thcse coherent structures do not have a characteristic scale but instead present a range of scales. Morcover, their spatial support decreases with scale and follows a power-law behavior before reaching the dissipative scales where thcir cores are locally regularized by dissipation. The spatial intermittency may then be explained by the gcometry of those cusp-like coherent structures. In addition Farge (1991b) supposes that each coherent structure may have a different scaling law and may begin to be dissipated at a different scalc than the others, which would thereforc correspond to the different peaks observed on the dissipative manifold $\operatorname{Re}(l, x)=1$ (see Farge et al 1990b). This conjecture scems consistent with Castaing's theory of turbulence (Castaing 1989, Castaing et al 1990), which, contrary to Kolmogorov's theory, assumes a possible weak dissipation in the inertial range. It is also consistant with Frisch and Vergassola's multifractal model of a possible intermediate dissipative range (Frisch \& Vergassola 1990). It may be that this geometrical interpretation of space intermittency, deduced from the wavelet analysis of two- and three-dimensional turbulent flows (Farge \& Rabreau 1988b, Farge et al $1990 \mathrm{a}, \mathrm{b}$ ) is wrong, resulting again from confusion between the scaling properties of the wavelet family and those of the turbulent field. As I have 


\section{FARGE}

previously said (Section 5.1), this is the most common pitfall encountered with wavelets and we are not yet immunized against it.

FOR TURBULENCE COMPUIING The objective is to be able to reduce the number of degrees of freedom necessary to compute the flow evolution, in order to simulate high Reynolds number flows. As we said in Section 1, for Fouricr spectral methods (Galerkin or pseudo-spectral) the number of degrees of freedom varies as $\mathrm{Re}$ in dimension two and $\mathrm{Re}^{9 / 4}$ in dimension three; the direct numerical simulations, i.e. without any ad hoc subgrid scale parametrization, are in this case limited to low Reynolds number flows, even with the fastest supercomputers available today. Fourier spectral methods are very precise if the flow remains regular. However, they are no longer appropriate when the flow develops shocks or steep gradients because solutions will then present some spurious oscillations everywhere in the domain (Gibbs' phenomenon). In this case we prefer to use finite element methods, or finite differences which may be thought as a particular case of finite element methods. In fact, wavelet bases are intermediate between finite element bases (due to their space localization) and Fourier bases (due to their scale localization). Now the point will be to use wavelets to develop new numerical techniques which will combine the advantages of both methods, while avoiding their inconveniences. Numerical methods using wavelets are presently in a nascent state, but seem very promising in the long term. For instance, in the case of partial differential equations, only Burger's equation in one dimension has been solved using wavelets in two different ways, a wavelet Galerkin method and also a wavelet particle technique.

To illustrate the principle of the wavelet Galerkin method, let us consider the one-dimensional evolution equation

$$
\left\{\begin{array}{l}
\frac{\partial u}{\partial t}+A(u)=0 \\
u(x, t=0)=u_{0}(x),
\end{array}\right.
$$

with $A$ as a differential operator. We can project (70) onto an appropriate multiresolution space $V$ yielding

$$
\left\langle\left[\frac{\partial \hat{u}}{\partial t}+A(\tilde{u})\right] \mid \phi\right\rangle=0,
$$

with $\phi$ ejther the scaling function (Latto \& Tencbaum 1990, Glowinski et al 1990) or the wavelet associated to the chosen multiresolution (Liandrat et al 1989, 1991; Liandrat \& Tchamitchian 1990; Maday et al 1990; Perrier 1989,1991 ). For a multiresolution based on the discrete filter $\hat{F}_{\phi}(k)$ 
(Section 4.2), the differentiation becomes very easy if we introduce a second filter $\hat{F}_{\phi}^{\prime}(k)$ which is the derivative of the first one, as proposed by Lemarie (Perrier 1991b). The only problems arise from the nonlinear operators, which presently must be computed in physical space at each time step, as is done for pseudo-spectral methods. In the case of $v \cdot \nabla v$-the nonlinear operator so important for turbulence-we can almost compute it directly in wavelet space (Beylkin 1991). The advantage of a wavelet Galerkin method, compared to Fouricr, is that it uses the lacunarity of the wavelet series and discards the wavelet coefficients below a certain threshold. Depending on the problem, we can then drastically reduce the number of degrees of freedom to compute. For instance, in the case of the onedimensional Burger's equation with a weak dissipation, Perrier has shown that 164 wavelet coefficients are sufficient to achieve the same precision of the solution, $\varepsilon=10^{-6}$, as with 1024 Fourier modes (Maday et al 1991; Perrier 1991a,b). In addition, she observes no Gibbs' phenomenon in the velocity ficld away from the shock, as is the case with the Fourier Galerkin method.

As we said in Section 5.1, turbulent flows may be thought of as a superposition of point vortices. This is the basic assumption of vortex numerical methods which is used for computing turbulent flows (Leonard 1985). In this case the solution is approximated as a finite sum of regularized Dirac masses, which evolve in amplitude and position. The same idea is behind the wavelet particle method, but in addition it allows the elementary vortices, substituted for the Dirac masses, to be deformed and therefore also to evolve in scale. The principle of the wavelet particle method (Basdevant et al 1990) is to look for an approximate solution of Equation (67), considered as a sum of a given number $N$ of wavelet particles $\Psi_{n}$, or wavelet atoms, which evolve in phase space according to:

$$
\left\{\begin{array}{l}
u(x, t)=\sum_{n=1}^{N} u_{n}(t) \psi \frac{x-x_{n}(t)}{l_{n}(t)}=\sum_{n=1}^{N} \Psi_{n}(x, t) \\
\Psi_{n}=\left\{u_{n}, I_{n}, x_{n}\right\}
\end{array}\right.
$$

with $l_{n}>0$ and $u_{n}, x_{n} \in \mathbf{R}$.

The double localization of wavelets in both space and scale ensures the independence, in the $L^{2}$ sense, of two wavelet particles $\Psi_{n}$ and $\Psi_{m}$ distant enough in phase-space, namely for

$$
\left|\frac{l_{m}}{l_{n}}-1\right| \gg 0 \text { and } \frac{\left|x_{m}-x_{n}\right|}{l_{n}} \gg 1 \text {. }
$$

Therefore the time evolution of the solution of Equation (67) will cor- 
respond to the trajectories of the wavelet particles in phase space. In regions where the solution is smooth there will be very few wavelet particles, whereas they will concentrate dynamically in regions where the solution may become singular. The wavelet particle method (also called "ondelettes mobiles") consists, for each time step, of finding the set of $\left\{u_{n}, \ldots, u_{n}, l_{1}, \ldots, l_{n}, x_{1}, \ldots, x_{n}\right\}$ that minimizes the local residual from Equation (67), where

$$
R=\int_{-\infty}^{+\infty}\left|\frac{\partial u}{\partial t}+A u\right|^{2} d x
$$

We must therefore solve a linear system of $3 N$ equations for $\partial u_{n} / \partial t, \partial l_{n} / \partial t$, and $\partial x_{n} / \partial t$, which is well conditioned only if the different wavelet particles remain sufficiently separated. How to handle wavclet-particle collisions is still an open problem; some tentative solutions are discussed in Perrier (1991a).

It is well-known that the importance of Fourier bases stems from the fact that they diagonalize differential operators. Although such operators lose this very simple form in a wavelet basis, due to the different frequencies involved in the wavelet and required by its space localization, they have a matrix realization that is almost diagonal (Meyer 1990a, Jaffard 1991d). Beylkin, Coifman, and Rokhlin have developed a very efficient algorithm, based on wavelets, which allows one to multiply a matrix by a vector very efficiently. Thus this algorithm is useful for diagonalizing or inverting certain classes of dense matrices, and is particularly appropriate to the case of integral operators (Beylkin et al 1991a). It uses the fact that wavelet coefficients decay rapidly in regions where the function is regular; after projecting $\mathscr{A}$ onto an orthogonal wavelet basis and reordering the terms, the BCR algorithm transforms $\mathscr{A}$ into a band matrix. For instance to solve $\mathbf{Y}=\mathscr{A} \mathbf{X}$, to accuracy $\varepsilon$, requires only $C(\varepsilon) \cdot N$ operations, with $C$ being a constant depending on the precision desired. This algorithm has been used to invert dense matrices of more than $2^{12}$ elements, which would have been impossible in practice with any other method. Other new algorithms, also based on wavelets, are presently being studied for inverting elliptic operators (Tchamitchian 1989a,b; Jaffard 1990b).

All these numerical methods based on wavelets are in a very preliminary phase and not yet ready to compute the Navier-Stokes or Euler equations. But, as a first step, we can still numerically solve these equations with other, more classical, algorithms and use wavelets only to locally filter the solutions or detect the strong gradient regions (such as shocks) in order to remesh the domain when and where it is necessary. Wavelets may also be used to define some new ways of forcing the flow field, for instance to 
excite only a given coherent structure and not the whole domain as with Fourier mode forcing. Finally, we can foresee using wavelets to develop new variants of the Large Eddy Simulation techniques, where the scale separation will no longer be defined in terms of Fourier wavenumbers, but will be based on a separation between coherent structures (i.e. very excited regions of wavelet phase space) which will be explicitly computed, while the background flow (i.e. regions of weak wavelet coefficients) will only be globally parameterized using some ad hoc model.

\section{CONCLUSION}

The present position of the founders of the wavelet theory-Morlet, Grossmann and Meyer-is to control the enthusiasm of the newcomers, who may overestimate the actual possibilities of the wavelet transform and then create some backlash effect resulting from their disappointment. My own view is that the wavelet transform is a very young technique which is evolving at a very fast pace, and we therefore must first become accustomed with it by performing extensive tests on well-known "academic signals" in order to develop a feeling for it before defining applications for which it will be useful.

We should also define some appropriate representations of the wavelet space, which is then critical for the two- and three-dimensional wavelet transform. We should develop a feel for the interpretation of wavelet coefficients, in particular for better understanding of the meanings of the modulus and phase in the case of complex-valued wavelets. In addition, we should test many different wavelets to try to optimize the choice of wavelet for a given problem. We should also find faster continuous wavelet transform algorithms. In the context of turbulence, the wavelet transform certainly opens some new possibilities, but we should not rely on it to solve the problem by chance, lest we run the risk - as has already heen the case in this field-of misinterpreting the results by mistaking the scaling behavior of the wavelet transform for the signal scaling. The wavelet transform is a sophisticated tool and its use might be very fruitful for the understanding of turbulence, if one is wise enough to first become accustomed with it.

To conclude I quote Yves Meyer (Meyer 1990c):

Wavelets are fashionable and therefore excite curiosity and irritation. It is amazing that wavelets have appeared, almost simultaneously in the beginning of the $80^{\circ} \mathrm{s}$, as an alternative to traditional Fourier analysis, in domains as diverse as speech analysis and synthesis, signal coding for telecommunications, (low-level) information extraction process performed by the retinian system, fully-developed turbulence analysis, renormalization in quantum field theory, functional spaces interpolation theory. . . But this 
pretention for pluridisciplinarity can only be irritating, as are all "great syntheses" which allow one to understand and explain everything. Will wavelets soon join "catastrophe theory" or "fractals" in the bazaar of all-purpose systems? It seems to me that "wavelets" have a slightly different position, since they do not constitute a theory but rather a new scientific tool. Indeed, they have never been used to explain anything. When $M$. Farge uses them to analyze turbulence simulation results, they play nearly the same role as the pair of glasses I use to read the "Apologie de Raimond Sebond." These glasses, now required by my age, should not be condemned if $I$ do nor understand Montaigne's thoughts, or glorified if I admire them. Likewise with wavelets whose modest, but essential, role is to help us to better study, at different scales, complex phenomena.

\section{ACKNOWLEDGMENTS}

The wavelet transform examples which illustrate this paper have been computed on the Cray-2 of the Centre de Calcul Vectoriel pour la Recherche, Palaiseau, and on other systems, in collaboration with JeanFrançois Colonna, Marc Duval-Destin, Eric Goirand, Philippe Guillemain, Matthias Holschneider, Gabriel Rabreau, and Victor Wickerhauser. I gratefully thank them for their help. I also thank very much all the members of the "wavelet community," who kindly agreed to read this paper and provide me with their comments. I am also very grateful to Laurette Tuckerman, Eric Gimon, and David Couzens for polishing my English.

\section{Literature Cited}

Antoine, J. P., Murenzi, R., Piette, B., Duval-Destin, M. 1990. Image analysis with 2D continuous wavelet transform: detection of position, orientation and visual contrast of simple objets. Se Meyer \& Paul I99I

Antoine, J. P., Carrette, P., Murenzi, R., Piette, B. 1991 . Image anaiysis with 2D continuous wavelet transform. In Wavelet Transforms and Multiresolution Signal Analysis, ed. 1. Daubechies, S. Mallat, A. Willsky, IEEE Trans. Inf. Theory. In press

Antoine, J. P., Duval-Destin. M. 1991. Local analysis of visual contrast with two dimensional wavelets. Inst. Phys. Théorique, Univ. Cathol. Louvain, Belgium. Preprint Argou, F., Arnéodo, A., Elezgaray. J.. Grasseau, G., Murenzi, R. 1988. Wavelet transform of fractal aggregatcs. Phys. Lett. $A$ 135:327

Argoul, F., Arnéodo, A., Grasseau, G., Gagne, Y., Hophinger, E., Frisch, U. 1989. Wavelet analysis of turbulence reveals the multifractal nature of the Richardson cascade. Nature 338(6210): $51-53$

Argoul, F., Arnéodo, A., Elezgaray, J., Grasseau, G., Murenzi, R. 1990. Wavelet transform analysis of the self-similarity of
Diflusion Limited Aggregate and electrodeposition clusters. Phys. Rev. A 41: 5537

Aristov, S. N., Frick, P. G. 1988a. Advective flows in plane rotating layers of conducting fluids. Magnetohydrodynamics 24(1): 13-20 (From Russian)

Aristov, S. N., Frick, P. G. 1988b. Largescale turbulence in a thin layer of nonisothermal rotating flujd. Fluid Dym. 23(4): 522-28 (From Russian)

Aristov, S. N., Frick, P. G. 1989. Large-scale turbulence in Rayleigh-Bénard convection. Fluid Dyn. 24(5); 43-48 (From Russian)

Aristov, S. N., Frick, P. G., Mikishev, A. B. 1989. Intcgral and local characteristics of large-scale turbulence in thin layers of fuids. On Turbulence, sth Europhys. Conf., Moscow, pp. 176-79

Aristov, S. N., Frick, P. G. 1990 . Nonlinear effects of interaction between the convective vortices and magnetic field in thin layer of conducting fluid. Magnetohydrodynamics 25(T): 82-88 (From Rus. sian)

Aristov, S. N., Frick, P. G., Mikishev, A. B. 1990. Appearance of large-scale structures in turbulent rotating layers of fluid. Int. 
Symp. Generation of Large-scale Structure in Continnous Media, Perm-Moscow, pp. 29-30

Arnéodo, A., Argoul, F.. Elezgaray, J., Grasseau, G, 1988. Wavelet transform analysis of fractals: applicalions to norequilibrium phase transitions. In Proc. Conf. Nonlinear Dynamics, Bologna, ed. G. Turchetti, p. 130. Singapore: World Scientific

Arnéodo, A., Grasseau, G., Holschneider, M. 1988. On the wavelet transform of multifractals, Phys. Rev. Lett. 61(20): $2281-84$

Arnèodo, A., Grasseau, G., Holschneider, M. 1989. Wavelet transform analysis of invariant measures of some dynamical systems. See Combes et al 1989, pp. 182-96

Arnéodo, A., Argoul, F., Grasseau, G. 1990. Transformation en ondelettes et renormalisation. See Lemarié $1990 \mathrm{a}$, pp. $125-91$

Améodo, A., Argoul, F., Bacry, E., Elezgaray, J., Freysz, E., Grasseau, G, Muzy, J. F, Pouligny, B. 1991. Wavelet transform of fractals: I. From the transition to chaos to fully developed turbulence. 11. Optical wavelet transform of fractal growth phenomena. See Meyer \& Paul 199!

Aubry, N., Holmes, P., Lumley, J. L., Stone, E. 1988. The dynamics of coherent structures in the wall region of a turbulent boundary layer. J. Fluid Mech. 192: I1573

Bacry, E.. Arnéodo, A., Frisch, U. Gagne. Y., Hopfinger, E. 1991. Wavelet analysis of fully developed turbulence data and measurement of scaling exponents. In Proc. Twrbulence 89: Organized Structures and Turbulence in Fluid Mechanics, Grenoble, Sept. 1989, ed. M. Lesieur, $\mathbf{O}$. Métais, pp. 203-15. Dordrecht: Kluwer

Basdevant, C., Legras, B., Sadourny, R., Béland. M. 1981. A study of barotronic model flows: intermittency, waves and prediclability. J. Atmos. Sci. 38: 2305-26

Basdevant. C.. Couder, Y. 1986. Experimental and numerical study of vortex couples in two-dimensional fows. J. Fluid Mech. 173: 225-51

Basdevant, C., Holșchneider, M., Perrier, V. 1990. Méthode des ondelettes mobiles. $C$. R. Acad. Sci. Paris, Sér. I: 647-52

Basseville, M., Benveniste, A. 1989. Multiscale statistical signal processing. $I E E E$ Int. Conference on Acoustics, Speech and Signal Processing, Glasgow, May 19894 : 2065-68

Bassevi]le, M., Benveniste, A. 1990. Multiscale autoregressive processes. Preprint No. 525, IRISA, Unjv. Beaulicu, Rennes, France
Battle, G. 1987. A block spin construction of Ondelettes, Part I: Lemarié functions. Commun. Math. Phys. ilo: 607-15

Battle. G. 1988. A block spin construction of Ondelettes, Part Il: The QFT Connection. Commun. Math. Phys, 1 14: 93-102

Bell. T., Nelkin, M. 1978. Time-dependent scaling relations and a cascade model of turbulence. J. Fluid Mech. 88(2); 36991

Benveniste, A. 1990. Multiscale signal processing: from QMF to wavelets. Preprint No. $550, I R I S A$, Univ. Beaulieu, Rennes, France

Benveniste, A., Nikoukhah, R., Willsky, A. 1990. Multiscale system theory. Preprint No. 5I 8 , IRISA, Univ. Beuulieu, Rennes, France

Benzi, R., Paladin, G., Parisi, G., Vulpiani, A. 1984. On the multifractal nature of fully developed turbulence and chaotic systems. J. Phys, A 17:3521--31

Benzi, R.. Vergassola, M. 1991. Optimal wavelet transform and its application to two dimensional turbulence. Fluid Dyn. Res. 8: 117-26

Beylkin, G., Coifman, R., Rokhlin, V. 199la. Fast wavelet transform. Comm. Pure Appl. Math. 44: 141-83

Beylkin, G., Coifman, R., Daubechies, I., Mallat, S., Meyer, Y., Ruskai, M. B., eds. 1991b. Wavelets and Their Applications. Boston: Jones \& Bartlett

Beylkin, G. 1991. On the represcritation of operators in bases of compactly supported wavelets. Schlumberger-Doll Research. Preprint

Bijaowi, A. 1991. Wavelets and astronomical image analysis. See Farge et al $1991 \mathrm{~b}$

Calderon, A. P. 1964. Intermediate spaces and interpolation, the complex method. Sind Math 24: [ 13-90

Castaing, B. 1989. Consequences d'un principe d'extremum en turbulence. $J$. Physique 50: 147-56

Castaing, B., Gagne, Y., Hopfinger, E. J. 1990. Velocity probability density lunctions of high Reynolds number turbulence. Physica $D$ 46: 177-200

Cohen, A., Daubechies, I., Feauveau, J. C. 1990. Biorthogonal bases of compactly supported wavelets. Comm. Pure Appl. Makh. Submitted

Cohen, A. 1990. Ondelettes, analyses multjrésolutions et traitement numérique du signal. PhD thesis. Univ, Paris, France

Coifman, R. R., Meyer, Y., Quake, $S_{\text {, }}$ Wickerhauser, M. V. i990a. Signal procossing and compression with wavelet packets. Dept. Math., Yale Univ, New Haven, CT. Preprint

Coifman, R. R., Meyer, Y., Wickerhauser, M. V. 1990b. Wavelet Packets. Dept. 
Math., Yale Univ., New Haven, CT. Preprint

Conbes, J. M., Grossmann, A., Tchamitchian, P., eds. 1989. Wavelets, Time-Frequency Methods and Phase Space. Ist Int. Wavelet Conf. Marseille, Dec. 1987, Inverse Probl. Theoret. Imaging. Springer

Dallard, T., Spedding, G. R. 1990. 2-D wavelet transforms. Aerosp. Dept., Univ. South. Calif., Los Angeles, CA. Preprint

Daubechies. I., Grossmann, A., Meyer, Y. 1986. Painless nonorthogonal expansions. J. Math. Phys. 27: 1271-83

Daubechies, I., Paul, T. 1987. Wavelets and applications. In Proceedings of the 8th International Congress of Mathematical Physics, ed. M. Mebkbout, R. Seneor. Singapore: World Scientific

Daubechies, I., Paul, T. 1988. Time frequency localization opcrators-a geometric phase-space approach: II The use of dilations. Inverse Probl. 4: 661-81

Daubechies, I. 1988. Orthonormal bases of compactly supported wavelets. Comm. Pure Appl. Moth. 4I(7): 909-96

Daubechies, I. 1989. Orthonormal bases of wavelets with tinite support-Connection with discrete filters. See Combes et al 1989, pp. $38-66$

Daubechies, 1. 1990. The wavelet transform. time-frequency localization and signal analysis. IEEE Trans. Inf. Theory 36:961

Daubechies. I. 1991. Ten lectures on wavelets. $C B M S$ Lecture Notes Series. SIAM

Daubechies, I., Mallat, S., Willsky, A. 1991. Wavelet transforms and multiresolution signal analysis. IEEE Trans. Inf. Theory. In press

Delprat, N., Escudié, B., Guillemain, P., Kronland-Martinet, R., Tchamitchian, P., Torresani, B. 1991. Asymptotic wavelei and Gabor analysis; extraction of instantaneous frequencies. See Daubechies et al 1991

Desnjanski, V. N., Novikov, E. A. 1974. Model of cascadc processes in turbulent flows. Appl. Math. Mech. 38(3): 507-13 (In Russian)

Dutilleux, P. 1989. An implementation of the algorithme à trous to compute the wavelet transform. See Combes et al 1989, pp. 298-304

Duval-Destin, M., Menu, J. P. 1989. Wavelet transform: a new basic spatial operator for visual psychophysics. Vision Res. Submitted

Duval-Destin, M., Murenzi, R. 1991. Spatiotemporal wavelet transform. Preprint $T R /$ VI/9 / $/ 49$, CERFACS, Toulouse, France

Duval-Destin, M., Muschietti, M. A., Torrésani, B. 1991. From continuous wavelets to wavelet packets. Preprint CPT, Cent.
Phys. Théorique, CNRS-Luminy, Marseille, France

Escudié, B., Torrésani, B. 1989. Wavelet analysis of asymptotic signals. Preprint CPT-89/P.2304, Cent. Phys. Théorique, CNRS-Luminy, Marseille, France

Esteban, D., Galand, C. 1977. Application of Quadrature Mirror Filters to split band voice coding schemes. Proc. 1977 IEEE Int. Conf. Acoustic, speech and signal processing, Hartford, pp. 191-95

Everson, R. M., Sirovich, L. 1989. A Survey of wavelet analysis applied to turhalence data. Report 89-182, Cent. Fluid Mech. Turbulence Comput., Brown Univ., Providence, RI

Everson, R., Sitovich, L., Srconivasan, K. R. 1990. Wavelet analysis of the turbulent jet. Phys. Lett. A 145: 314

Falconer, K. J. 199t. Wavelets, fractals and order-two densities. See Farge et al $1991 b$

Farge, M., Rabreau, G. 1988a. Wavelet transform to analyze coherent structures in two-dimensional turbulent flows. Proc. Scaling, Fractals and Nonlinear Variability in Geophysics 1, Paris, June 1988

Farge, M., Rabreau, G. 1988b. Transformée en ondelettes pour détecter et analyser les structures cohérentes dans les écoulements turbulents bidimensionnels. C. R. Acad. Sci. Paris Ser. II 307: 1479-86

Farge, M., Sadourny, R. 1989. Wave-vortex dynamics in rotating shallow water, $J$. Fluid Mech. 206: 433-62

Farge, M., Holschneider, M. 1989. Twodimensional wavelet analysis of twodimensional turbulent fows. Proc. Scaling, Fractals and Nonlinear Variability in Geophysics II, Barcelone, March 1989

Farge, M., Holschneider, M., Colonna, J. F. 1990a. Wavelet analysis of coherent structures in two-dimensional turbulent flows. In Topological F/uid Mecharices, ed. H. K. Moflatt, pp. 765-76. Cambridge: Cambridge Univ. Press

Farge, M. 1990. Transformée en ondelettes continuc et application à la turbulence. Joum. Amu. Soc. Math. France, May 1990: $17-62$

Farge, M., Iolschneider, M. 1991. Interpretation of two-dimensional turbulence energy spectrum in terms of quasi-singularity in some vot tcx cores. Europhys. Lett. 15(7): 737-43

Farge, M., Guezennec, Y., Ho, C. M., Meneveau, C. $1990 \mathrm{~b}$. Continuous wavelet analysis of coherent structures. Proc. Summer Prog. Cent. Turbulence Res., Stanford Univ. NASA-Ames, Stanford, CA

Farge, M. 199 ta. Continuous wavelet transform application to turbulence. See Beylkin et al $199 \mathrm{lb}$

Farge, M. 1991b. Lévolution des théories de 
la turbulence. To appear in Chaos et Déterminisme, Collection Points-Sciences, Lc Scuil

Farge, M., Holschneider, M., Philipovitch, T. I99la. Formation et stabilité des quasisingularités $\mathrm{t}^{-1 / 2}$ en turbulence bidimensionnelle. C. R. Acad. Sc, Paris, Submitted

Farge, M., Hunt, J., Vassilicos, C., eds. 199 Jb. Watelets, Fractals and Fourier Transform: New Developments and New Applications, Oxford: Oxford Univ. Press. In press

Farge, M., Goirand, E., Wickerhauser, V. 199 lc. Wavelet packcts analysis, compression and filtering of two-dimensional turbulent flows. LMD, Ecole Normalé Supérieure, Paris. Preprint

Feauveau, J. C. 1989. Filtres micoirs conjugués: une théorie pour les filtres miroirs en quadralure et l'analyse multirésolution par ondelettes. C. R, Acad. Sci. Paris, Sér. II 308: 1479_86

Feauveau, J. C. 1990. Analyse multirésolution par ondelettes. C.R. Acad. Sci. Paris, Ser. / 309: 853-56

Flandrin, P. 1988. Tinte-frequency and timescale. IEEE Fourth Annu. ASSP Workshop Spectrum Estimation and Modeling, Minneapolis, August 1988, pp. 77-80

Flandrin, $P$. 1990. Wavelets and related time-scale transforms. SPIE Proc. Advanced Signal Processing Algorithms, Architectures and Implementations, ed. F. T. Luk, 1348: 2-โ3

Flandrin, $P_{+}$Rioul, O. 1990. Aftine smoothing of the Wigner-Ville distribution. IEEE ICASSP Conference, Albuquerque, April 1990 , pp. $2455-58$

Flandrin, P. 1991a. Wavelet analysis and synthesis of fractional Brownian motion. In Watelet Transforms and Muliresolution Signal Analysis, ed. 1. Daubechies, S. Mallat, A. Willsky, IEEE Trans. Inf. Theory, spccial issue

Flandrin, P. 199!b. Fractional Brownian motion and wavelets. See Farge et al 1991 b

Freysz, E., Pouligny, B., Argoul, I', Arnéodo, A. 1990 . Optical wavelet transform of fractal aggregates. Phys, Rev. Lett. 64: 745

Frick, P. G. 1983. Hierarchical model of two-dimensional Iurbulence. Magnetohydrodynamics 19(1): $48-53$ (From Russian)

Frick, P. G. 1984. Two-dimensional MHD. turbutence: Hierarchical model, Magnetohydrodynumics 20(3) (Frum Russian)

Frick, P, G. 1986a. A cascade model of twodimensional turbulent convection. Fluid Mech. Sov. Res. 15(2): 80-92 (From Russian)

Frick, P. G. 1986b. Modeling of cascade pro- cess in two-dimensional turbulent convection. J. Appl. Mech. Tech. Phys. 27(2): 221-28 (From Russian)

Frick, P. G. 1987. Modeling of the spacetime structure of developed two-dimensional turbulent convection. Fluid Mech. Sov. Res. [6(3): 49-54 (From Russian)

Frick, P. G., Mikishev, A. V. 1990. Investigation of local and integral characteristics of developed two-dimensional turbulence using the hierarchical model. Second World Congr. Compur. Mech., August 1990, Stuttust

Frick, P. G., Zimin, V. D. 1991. Hierarchical models of turbulence. Sce Farge et at $199 \mathrm{lb}$

Frisch, U., Sulem, P. L. 1975. Remarqule sur la multiplication dans les espaces de Sobolev et application aux équations d'Euler d'un fuide illimité. C. R. Acad. Sci. Paris, Sér. A 280: 1117-20

Frisch, U., Sulem, P. L., Nelkin, M. 1978. A simple dynamical model of intermittent fully developed turbulence. $J$. Fluid $M$ ech. 87: 719-36

Frisch, U., Vergassola, M. 1990. A prediction of the multifractal model; the intermediate dissipation range. Europhys. Letf. Submitted

Gabor, D. 1946. Theory of communication. J. Inst. Electr. Eng. 93(III): 429-57

Gasquet, C., Witomski, P. 1990. Analyse de. Foturiet et Applications: Filtragre, Calcul Nimerique, Ondeleftes. Paris: Masson

Ghez, J. M., Vaienti, \$. 1989. On the wavelet analysis for multifractal sets. J. Stat. Phys. $57(12): 415-20$

Gledzer, E. B, Dolzhansky, F. B., Obukhov, A. M. 1981. Systems of hydrodynamical type and their applications. $C, R, A c a d$. Sci., Moscow (In Russian)

Glowinski, R., Lawton, W. M., Ravachol, M., Tenenboum, E. 1990 . Wavelet solution of linear and nonlinear elliptic, parabolic and hyperbolic problems in one space dimension. $9 \mathrm{~h}$ Int. Conf. Appt. Sci. Eng., SIAM, Philadelphia

Goupillaud, P., Grossmatn, A., Morlet, J. 1984. Cycle-octave and related transforms in seismic signal analysis. Gevexploration 23: $85-105$

Grossmann, A. 1988. Wavelet transforms and edge detection. Slochastic Processes in Physics and Engineeriny, ed. P. Blanchard, L. Streit, M. Hazewinket. Boston: Reidel

Grossmann, A., Morlet, J. 1984. Decomposition of Hardy functions into square integrable wavelets of constant shape. $S I A M$ J. Math. Anal. 15(4): 723-36

Grossmann, A., Paul, T, 1984. Wave functions on subgroups of the group of affine canonical transformations. Resonances, 


\section{FARGE}

Models and Phenomena, Lecture Notes in Physics, No. 21I. Herlin: Springer-Verlag

Grossmann, A., Morlet, J. 1985. Decomposition of functions into wavelets of constant shape, and related transforms. In Mathematics + Physics, Lectures on Recent Results, ed. L. Streit, 1: 135-65. Singapore: World Scientific

Grossmann, A., Morlet, J., Paul, T, 1985. Transforms associated to square integrable group representations I: general results. J. Math. Phys. 26: 2473-79

Grossmann, A., Morlet, J., Paul, T, 1986. Transforms associated to square integrable group representations II: examples. Ann. Inst. Henri Poincaré. Phys. Théorique 45: $293-309$

Grossmann, A., Holschneider, M., Kronland-Martinet, R., Morlet, J. 1987. Detection of abrupt changes in sound signals with the help of wavelet transforms. In Advances in Electronics and Electron Physics, Suppl. 19, Inverse Problems, pp. 289306. Academic

Grossmann, A., Kronland-Martinent, R. 1988. Time-and-scale representations obtained through continuous wavelet transforms. In Signal Processing IV: Theories and Applicalions, EURASIP 88, ed. J. L. Lacoume, A. Chehikian, N. Martin, J. Malbos, pp. 675-82. Amsterdam: Elsevier

Grossmann, A., Kronland-Martinct, R., Morlet, J. 1989. Reading and understanding continuous wavelet transforms. See Combes et al 1989, pp. 220

Guillemain, P. 1991. Application of wavelet analysis in NMR spectroscopy. See Meyer \& Paul 1991

Haar, A. 1909. Zur Theorie der Orthogonalen Funktionensysteme. Gottingen, Juli 1909

Holschneider, M. 1988a. L'analyse d'objets fractals et leur transformée en ondelettes. PhD thesis. Univ. d'Aix-Marseille II, Luminy, France

Holschneider, M. $1988 \mathrm{~b}$. On the wavelet transformation of fractal object. J. Stat. Phys. 50: 953-93

Holschncider, M., Kronland-Martinet, R., Morlet, J., Tchamitehian, P. 1988. The "algorithme à trous." IEEE Trans. ASSP. Submitted

Holschneider, M., Kronland-Martinet, R, Morlet, J., Tchamitchian, P. 1989. A realtime algorithm for signal analysis with the help of the wavelet transform. See Combes et al 1989 , op. $286-97$

Holschneider, M., Tchamitchian, P. 1989. Pointwise analysis of Riemann's nondifferentiable function. Inventiones Math. Submitted

Holschneider, M. 1990. Inverse Radon transform through inverse wavelet transtorm. Inverse Probl. Submitted

Holschneider, M., Tchamitchian, P. 1990. Régularité locale de la fonction "nondiflërentiable" de Riemann. See Lemarié 1990 a, pp. 102-24

Holschneider, M. 1991. Watelet Analysis. Textbook submitted to Springer

Jaffard, S. 1989a. Exposants dc Hölder on des points donnés et coefficients d'ondelettes. C. R. Acad. Sci. Paris, Sér. I 308: 79-8I

Jafiard, S. $1989 \mathrm{~b}$. Construction of wavelets on open sets. See Combes et al 1989, pp. 247-52

Jafiard, S., Meyer, Y. 1989. Bases d'ondelettes dans des ouverts de $\mathbf{R}^{n}$, J. Math. Pures Appl. 68: 95-108

Jafiard, S. 1990a. Construction et propriétes des bases d'ondelettes. Remarques sur la contrôlabilité exacte. PhD thesis. l'Ecole Polytechniquc, Palaiseau. France

Jaflard, S. 1990b. Wavelet methods for fast resolution of elliptic problems. Preprint $90 / 5$, Lab. Math. Modélisation, Ecole Nat. Ponts-et-Chaussees, La Courtine, Noisyle-Grand, France

Jaffard, S. I99/a. Detection and identification of singularities by the continuous wavelet transform. Preprint, Lab. Math. Modélisation, Ecole Nat. Ponts-et-Chaussees, La Courtine, Noisy-le-Grand, France

Jaffard, S. $1991 \mathrm{~b}$. Orthonormal and continuous wavelet transform: algorithms and applications to the study of pointwise properties of functions. See Fargc et al $1991 \mathrm{~b}$

Kim, J,, Moin, P., Moser, R. 1987. Turbulence statistics in fully developed channel flow at low Reynolds number. $J$. Fluid Mech. 177: 133-66

Kline, S. J., Reynolds, W. C., Schraub, F. A., Runstadler, P. W. 1967. The structure of turbulent boundary layers. J. Fluid Mech. 30(4): 741-73

Kolmogorov, A. M. 196I. A refinement of previous hypotheses concerning the local structure of turbulence in viscous incompressible fluid at high Reynolds number. J. Fluid Mech. 177: 133-66

Kraichnan, R. H. 1974. On Kolmogorov's inertial-range theories. I. Fluid Mech. 62: 305-30

Kronland-Martinet, R., Morlet, J., Grossmann, A. 1987. Analysis of sound patterns through wavelet transforms. Int. J. Pattern Anal, Artif. Intell. 1(2): 273302

Kronland-Martinet, R. 1988. The use of the wavelet transform for the analysis, synthesis and processing of speech and music. sounds. Comput. Music J. MIT Press, 12: 4 
WAVELET TRANSFORMS

Latto, A., Tenenbaum, E. 1990. Compactly supported wavelets and the numerical solution of Bürgers equation. C. R. Acad. Sci. Paris, Sér. I 311: $903-9$

Laufer, J. 1975. New trends in experimental turbulence research. Antur. Rev. Fluid Mech. 7: 307-26

Lemarié, P. G., Meyer, Y. 1986. Ondelettes et bases hilbertiennes. Rev. Mat. Bbero Am. 2: $1-18$

Lemarié, P. G. 1988. Ondelettes à localisation exponentielle. J. Math. Pures Appl. 67: $227-36$

Lemarié, P. G., ed. 1990a. Les Ondelettes en 1989. Lecture Notes in Mathematics, Berlin: Springer

Lemarié, P. G. 1990b. Introduction à la théorie des ondelettes. See Lemarié 1990a $1-13$

Lemarié, P. G. 1990c. Analyse multi-échelles et ondelettes à support compact. See Lemarjé 1990a, pp. 26-38

Leonard, A. 1985. Computing three-dimensional incompressible flows with vortex elements. Annu. Rev. Fluid Mech. 17:52359

Liandrat, J., Perrier, V, Tchamitchian, P. 1989. Numerical resolution of the regularized Bürgers equation using the wavelet 1ransform. See Meyer \& Paul 1991

Liandrat, J., Tchamitchian, P. 1990. Resolution of the ID regularized Bürgers equation using a spatial wavelet approximation-Algorithm and numerical results. ICASE Report, NASA Langley, Hampton, Virginia

Liandrat, J., Moret-Bailly, F. 1990. The wavelet transform: some applications to fuid dynamics and turbulence. Eur. $J$. Mech. B/Finids 9(1): 1-19

Liandrat, J., Perrier, V., Tchamitchian, P. 1991. Numcrical resolution of nonlinear partial differential equations using the wavelet transform. See Beylkin et al $199 \mathrm{lb}$

Lumley, J, L. 1981, Coherent structures in turbulence. In Transition and Turbulence, ed. R. E. Meyer, pp. 215-4I. New York: Academic

Maday, Y., Perrier, V., Ravel, J. C. 1990. Adaptativité dynamique sur bases d'ondelettes pour l'approximation d'equations aux dérivées partielles. C. $R$. Acod. Sct. Paris. Submitted

Mallat, S. 1988. Review of multifrequency channel decompositions of images and wavelet models. In Acoustic, Speech and Stgnal Processing, Mullidimensional Signal Processing, IEEE (special issue)

Mallat, S. 1989a. A thcory for multiresolution signal decomposition: the wavelel representation. IEEE, Trans. on Patlem Anal. Machine Intell. 2: 7
Mallat, S. $1989 \mathrm{~b}$. Multiresolution approximations and wavelet orthonormal bases of $L^{2}(\mathbf{R})$. Trans, Am. Math. Soc. 315; 6987

Mallat, \$. $1989 \mathrm{c}$. Multiresolution approximation and wavelets. Report GRASP Lab., Dep1. Comput. Inf. Sci., Univ. Pennsylvania, Philadelphia, PA

Mallat, S. $1989 \mathrm{~d}$. Multiresolution approach to wavelets in computer vision. See Combes et al 1989. (2nd ed. 1990, p. 313)

Mallat, S., Hwang, L. H. 1990. Singularity detection and processing with wavelets. Couramt Inst. Tech. Rep. No. 549, New York, NY

Mallat, S., Zhong, S. 1990. Complete signal representation with multiscale edges. Courant Inst. Tech. Rep. No. 483, New York, NY

Mallat, S., Zhong, S. 1991. Wavelet transform maxima and multiscale edges. See Beylkin et al 1991b

Mandejbrot, B. 1974. Intermittent turbulence in sel[-similar cascades: divergence of high moments and dimension of carrier. J. Fluid Mech. 62: 331-58

McWilliams, J. 1984. The emergence of isolated coherent vortices in turbulent flow. J. Fluid Mech. 146: 2I-433

Meneveau, C. 1991 a. Analysis of turbulence in the orthonormal wavelet representation. $J$. Fluid Mech. In press

Meneveau, C. $1991 \mathrm{~b}$. Wavelet analysis of turbulence: the mixed energy cascade. See Farge et al $1991 \mathrm{~b}$

Meneveau, C. 1991. Dual spectra and mixed energy cascade of turbulence in the wavelet representation. Phys. Rev. Left. 66: 1450

Meyet, Y. 1985. Principe d'incertitude, bases hilbertiennes et algèbres d'opérateurs. Séminaire Bourbaki, 1985-86, 662, Astérisque, Société Mathématique de France, Paris, France

Meyer, Y. 1986. Ondelettes et fonctions splines. Séminaire Equations aux dérioées partielles, Cent. Math. Ecole Polytech., Palaiseau, France

Meyer, Y. 1987a. Ondelettes, fonctions splines et analyse graduée. Rapport CEREMADE, No. 8703, Univ. ParisDauphine, Paris. France

Meyer, Y. $1987 \mathrm{~b}$. Wavelets and operators. Rapport CEREMADE, No. 8704, Univ. Paris-Dauphine, Paris, France

Meyer, Y. 1988. Orthonormal wavelets. Congr. Int. Phys. Math, Swansea, July.

Meyer, Y. 1989a. Ondelettes, filtres miroirs en quadrature traitement numérique de l'image. Gaz. Math, 40: 31-42

Mcyer, Y. 1989 b. Orthonormal wavelets. Set Combes et al 1989, pp. 21-37

Meyer, Y. 1989c. Ondelettes, filtres miroirs 
en quadratuse et traitement numérique de l'image. See Lemarié 1990a, pp. 14-25

Meyer, Y. 1990k. Ondelettes et opérateurs, tome I. Actualités Mathématiques. Paris: Hermann

Meyer, Y. 1990b. Le calcul scientifique, les ondelettes et les filtres miroirs en quadrature. Rapport CEREMADE, No. 0007 , Univ. Paris-Dauphine, Paris, France

Meyer, Y. 1990c. Ondelettes et applications. J. Annu. Soc. Math. France, May 1990, Soc. Française Math., Paris, France, pp. $1-15$

Meyer, Y., Paul, T., eds. 1991. Wavelets and Applications. Procetings of the Second International Wavelet Conference, Mar. seille, June 1989. Paris: Masson. In press

Mikishev, A. V., Frick, P. G. 1989. Intermittence in ideal two-dimensional magnetohydrodynamic turbulence. Magnetohydrodynamics 25(1): 127-30 (From Russian)

Mikishev, A. Y., Frick, P. G. 1990. On the spectral laws in two-dimensional turbulent flow with linear friction. Magnetohydrodynamics 26(1): 136-39 (From Russian)

Moret-Bailly, F., Chauve, M. P., Liandrat, J., Tchamitchian, P. 199 I. Determinations du nombre de Reynolds de transition dans utle étude de couche limite sur un disque toumant. C. R. Acad. Sci. Paris. Submitted

Morlet, J. 1981. Sampling theory and wave propagation. Proc. 5/st Annu. Meet. Soc. Explor. Geophys, Los Angeles

Morlet, J. 1983. Sampling theory and wave propagation. NATO ASI, Vol. FI, Issues on Acoustic Signal/Image Processing and Recognition, ed. C. H. Chen. Berlin: Springer

Murenzi, R. 1989. Wavelet transforms associated to the $\mathrm{N}$-dimensional Euclidean group with dilatations: signal in more than one dimension. See Combes et al 1989, pp. 239-46

Murenzi, R. 1990. Ondelettes mullidimensionnelles et application à l'analyse d'ımages. PhD thesis. Univ. Cathol. Louvain, Louvain-la-Neuve, Belgium

Muzy, J. F, Bacry, E., Arnéodo, A, 1991. Wavelets and multifractal formalism for singular signals. Phys. Ret. Lett. Submitted

Nakano, T. 1988. Direct interaction approximation of turbulence in the wave packet representation. Phys. Fltids 31: 1420

Nguyen Duc, J., Sommeria, J. 1988. Experimental characterization of steady twodimensional vortex couples. $J$. Fhid Mech. 192: 175-92

Otaguro, T., Takagi, S, Sato, H. 1989. Pat- tern search in a turbulent signal using wavelet anaiysis. Proc. 21st Japan Symp. on Turbulence. Tokyo, Japan

Parisi, G., Frisch, U. 1985. Fully developed turbulence and intermittency. In Proc. Insernational School on Turbulence and Predictability in Geophysical Fluid Dynamics and Climate Dynamics, ed. M. Ghil, R. Benzi, G. Parisi, pp. 71-88. Amsterdam: North-Holland

Paul, T. 1984. Functions analytic on the halfplane as quantum mechanical states. $J$. Math. Phys. 25: 11

Paul, T. 1985a. Affine coherent states and 1)e radial Schröodinger equaljont. Anm. Inst. Henri Poincaré. Submitted

Paul, T. 1985b. Ondelettes et mécanique quantique. PhD thesis. Univ. Aix-Marseille lI, Luminy, Marseille. France

Paul, T. 1986. A characterization dilation analytic operators. In Schrödinger operators, Aarhus, 1985, Lecture Notes in Mathematics, 1218. Berlin: Springer

Paul, T. 1989. Wavelets and path integrals. See Combes et al 1989, pp. 204-8

Perrier, V. 1989. Toward a method for solving Partial Differential Equations using wavelet bases. See Combes et al 1989, pp. 269-82

Perrier, V., Basdevant, C. 1989. La décomposition en ondelettes périodiques, un outil pour l'analyse de champs inhomogènes. Théorie et algorithmes. La Recherche Aérosp. 3: 53-67

Perrier, V. $199 \mathrm{la}$. Ondelettes et simulation numérique. PhD thesis. Univ. Paris VI, Paris, France

Perrier, V. 1991b. Apport des ondelettes à la résolution numérique d'équations aux dérivées partielles. J. Soc, Moth. Appl Ind. In press

Qian, J. 1988. Cascade model of turbulence. Phys. Fluids 31(10): [865-74

Rioul, O. 1987. Ondelettes et traitement du signal. Rapport d'option de Mathématiques, Ecole Polytech., Palaiseau, France

Rioul, O. 1991. A unifying multiresolution theory for the discrete wavelet transform, regular filter banks and pyramid iransforms. IEEE Trans. Acoustics, Speech and Signal Processing. Submitted

Saracco, G., Tchamitchian, P. 1988. A study of acoustic transmission of transient signal in an inhomogeneous medium with the help of the wavelet transform-Application to an air-water plane interface. In Electromaynetic and Acoustic Scallering: Detection and Inverse Problem, ed. C. Burrely, P. Chiapetta, B. Torrésani. Marseille: World Scientific

Saracco, G., Grossmann, A., Tchamitchian, P. 1989. Use of wavclet transforms in the 
study of propagator of transient acoustic signals across a plane interface between two homogenenus media. See Combes et al 1989, pp. ! 39-46

Stromberg, J. O. 1981. A modified Haar system and higher order spline systems. Conf. Harmonic Analysis in Honor of Antomi Zygmund, II, ed. W. Becker et al, pp. 47593. Wadworth math. series

Tchamitchian, P. 1986. Calcul symbolique sur les opérateurs de Calderon-Zygmund et bases inconditionnelles de $L^{2}(\mathbf{R})$. C. $R$. Acad. Sci. Paris, Sér. $1303: 6$

Tchamitchian, P. 1987. Biorthogonalité et théorie des opérateurs. Rev. Math. Iberoam. 3: 2

Tchamitchian, P. 1989a. Bases d'ondelettes et intégrales singulières: analyse des fonctions et calcul sur les operateurs. These d'habilitation d diriger des recherches. Univ, Aix-Marseille II, Luminy, Marseille, France

Tchamitchian, P. 1989b. About wavelets and elliptic operators. See Combes et at 1989, pp. 263-68

Tchamitchian, P. 1989c. Ondelettes ct intégrale de Cauchy sur les courbes lipchitziennes. Ann. Math. 129: 641

Tchamitchian, P., Torrésani, B. I991. Kidge and skeleton extraction from the wavelet transform. See Beylkin 199tb

Tennekes, H., Lumley, J. L. 1972. A First Course in Turbulence. Cambridge: MIT Press

Torrésani, B. 1991. Tìme-frequency representations: wavelet packets and optimal decomposition. Ann. Inst. H. Poincaré, Phys. Theorique. In press

Townsend, A. A 1956. The Structure of Turbulent Shear Flow. Chapter 6. Cambridge: Cambridge Univ. Press

\section{References added in proof}

Fargc, M. 1991c. Wavclets and turbulence. INSU Summer School on Geophys. Fluid Dyn., July 1991, Rescoff
Vergassola, M., Frisch, U. 1990. Scaling exponents for global and local self-similar processes. Physica D. Submitted

Vetterli, M., Herley, C. 1990a. Wavelets and filter basks: theory and design. IEEE Trans. Acoustics, Speech Signal Processing. Subtnitted

Vetterli, M.,Herley, C. $1990 \mathrm{~b}$. Wavelets and filter banks: relationship and new results. IEEE Trans. Aconstics, Speech Signal Processing. In press

Vetterli, M., Herley, C. $1990 \mathrm{c}$. Linear phase wavelets. IEEE Truns. Acoustics, Speech Signal Processing. In press

Wickerhauser, M. V. 1991. INRIA lectures on wavelet packet algorithms. Preprint, Dept. Math., Yale Univ., New Haven, CT

Yamada, M., Ohkitani, K. 1990a. Orthonormal wavelet expansions and its application to turbulence, Prog. Theor. Phys. 83: 819

Yamada, M., Onkitani, K. 1990b. Orthonormal wavelet analysis of turbulence. Preprint. Res. Inst. Math. Sci., Kyoto Univ., Kyoto, Japan

Zimin, V. D. 1981. Hierarchical model of turbulence. Izo. Atmos. Ocean Phys. 17: 941-49 (From Russian)

Zimin, V. D., Frick, P. G., Shaidurova, T. E. 1986 . Hierarchical bases for turbulent fields describing. Preprint, Inst. Continuous Media Mech. Korolyov I, Perm, Soviet Union (In Russian)

Zimin, V. D., Frick, P. G. 1988. Turbulent Convection. Moscow: Nauka (In Russian)

Zimin, V. D. 1990a. Hierarchy model of turbulence. $6 \mathrm{th}$ Beer-Sheva Int. Sem. MHD Flows Turbulence, Jerusalem

Zimin, V. D. 1990 b. Hierarchy model of turbulence. Imt. Symp. Generation of largescale struchure in continuous media, PermMoscow, June, p. 263

Granlund, G. H. 1978. In search of a general picture processing operator. Comput. Graphics Image Process. 8: 15\$-73 